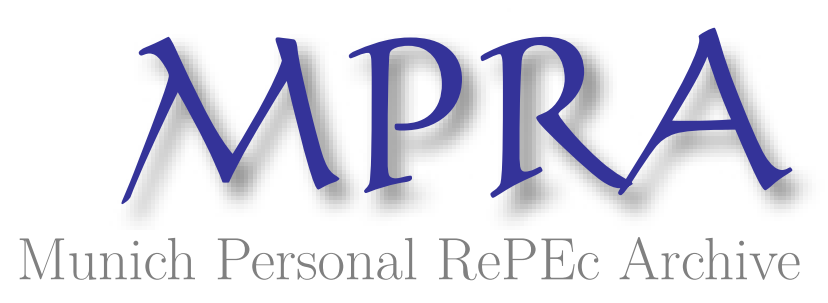

\title{
Static Portfolio Choice under Cumulative Prospect Theory
}

Bernard, Carole and Ghossoub, Mario

University of Waterloo

17 January 2009

Online at https://mpra.ub.uni-muenchen.de/16230/

MPRA Paper No. 16230, posted 13 Jul 2009 23:43 UTC 


\title{
Static Portfolio Choice under
}

\section{Cumulative Prospect Theory}

\author{
Carole Bernard* and Mario Ghossoub ${ }^{\dagger \ddagger}$ \\ University of Waterloo
}

\begin{abstract}
We derive the optimal portfolio choice for an investor who behaves according to Cumulative Prospect Theory. The study is done in a oneperiod economy with one risk-free asset and one risky asset, and the reference point corresponds to the terminal wealth arising when the entire initial wealth is invested into the risk-free asset. When it exists, the optimal holding is a function of a generalized Omega measure of the distribution of the excess return on the risky asset over the risk-free rate. It conceptually resembles Merton's optimal holding for a CRRA expected-utility maximizer. We derive some properties of the optimal holding and illustrate our results using a simple example where the excess return has a skew-normal distribution. In particular, we show how a Cumulative Prospect Theory investor is highly sensitive to the skewness of the excess return on the risky asset. In the model we adopt, with a piecewise-power value function with different shape parameters, loss aversion might be violated for reasons that are now well-understood in the literature. Nevertheless, we argue, on purely behavioral grounds, that this violation is acceptable.
\end{abstract}

Key-words: Cumulative Prospect Theory, Portfolio Choice, Behavioral Finance, Omega Measure.

JEL codes: D81, G11, D03.

*c3bernar@uwaterloo.ca, tel: +1 5198884567 ext:35505

†'ghossoub@umich.edu, tel: +1 5198884567 ext:33835

${ }^{\ddagger}$ We would like to thank Phelim Boyle, Virginia Young and Harry Panjer for helpful discussions. Both authors acknowledge support from the Natural Sciences and Engineering Research Council of Canada. 


\section{Introduction}

The present work is concerned with optimal investment under Cumulative Prospect Theory. In its original version (Kahneman and Tversky (1979)), Prospect Theory violated first-order stochastic dominance. Adopting Quiggin (1982)'s idea of distorting cumulative probabilities of ranked outcomes instead of individual probabilities resolved this issue and led to Cumulative Prospect Theory (Tversky and Kahneman (1992)). Although this theory was published many years ago, few theoretical work has been done. Empirical research ${ }^{1}$ has been testing Cumulative Prospect Theory and providing evidence of its relevance for explaining individuals' decision making ${ }^{2}$. The object of the present paper is to examine the very natural question of how an investor optimizes her portfolio holding in a risky asset under Cumulative Prospect Theory, in a one-period economy with one risk-free asset and one risky asset. The problem is simple but the results are new and interesting. This question has already been extensively studied under Expected Utility Theory ${ }^{3}$.

We show that there is a strong relationship between Omega performance measures and the optimal holding of an investor under Cumulative Prospect Theory (CPT) with piecewise-power value function with different shape parameters, under a suitable choice of the status quo. We study the properties of this optimal holding and discuss how sensitive an investor behaving according to CPT is to skewness, asymmetric distributions and curvature of the value function.

Measures of performance have evolved tremendously since the pioneering work of Markowitz and Sharpe. The mean-variance framework is certainly the most well-known investment decision rule. Recently, however, a significant amount of research has been done on more complex performance measures, and new performance measures such as the Omega measures (Keating and Shadwick (2002)) or the gain-loss ratio of Bernardo and Ledoit (2000) are becoming popular. The links between Cumulative Prospect Theory and risk measures have already been studied by Jarrow and Zhao (2006) and De Giorgi, Hens and Mayer (2006). The former utilize the lower partial moment as a risk measure for downside loss-aversion, while the latter develop a behavioral risk-reward model based on the gain-loss trade-off in Cumulative Prospect Theory.

At the same time, Expected Utility Theory is still today the most widely

\footnotetext{
${ }^{1}$ See for instance Camerer, Loewenstein and Rabin (2004).

${ }^{2}$ One reason for the lack of theoretical research is the complexity of the setup of Cumulative Prospect Theory. Indeed in Cumulative Prospect Theory (CPT), the value function is neither concave nor convex and probabilities are distorted. Convex analysis is the main tool in Expected Utility Theory and cannot be used in CPT.

${ }^{3}$ See Gollier (2001), Chapter 4 for a review of some of these results.
} 
used model to explain individuals' decision making under uncertainty. However empirical evidence of systematic violations of Von Neumann and Morgenstern's axioms of Expected Utility Theory - notably the independence axiom - led many economists to consider alternative models of choice whereby the economic agent is not assumed to be the usual rational decision maker he has been so far ${ }^{4}$. These findings resulted notably in three major alternatives to Expected Utility Theory, namely Prospect Theory (Kahneman and Tversky (1979)), the Rank-dependent Utility Theory (Quiggin (1982) and Schmeidler (1989)) and the Dual Theory of Choice (Yaari (1987) $)^{5}$. In this paper, we work under Cumulative Prospect Theory and highlight the link between the new performance measures and this new framework to model the behavior of economic agents.

Some research has already been done on optimal investment under Cumulative Prospect Theory (CPT). Most of the previous work takes place in a dynamic setting or when no probability distortion exists. The optimal portfolio choice problem for a loss-averse investor is solved by Berkelaar, Kouwenberg and Post (2004) (in a complete market but where no distortion is applied to the probabilities). Gomes (2005) also studies the optimal portfolio choice of a loss-averse investor and shows that there exists a certain wealth level above which the investor follows a portfolio insurance rule. Benartzi and Thaler (1995) show that loss aversion might explain the equity premium puzzle. De Giorgi, Hens and Levy (2004) show that under the assumption of normality of returns in CPT, the CAPM still holds when market equilibria exist. However, equilibria do not always exist due to the so-called infinite short-selling problem. De Giorgi and Hens (2006) solve this problem by modifying the form of the value function, using a piecewise negative exponential function. De Giorgi, Hens, and Rieger (2008) examine an alternative solution to the infinite short-selling problem by imposing short sale constraints in the presence of heterogeneity of beliefs. They show that in this case, even though the infinite short-selling problem is solved, non-existence of equilibria may be caused by discontinuities in the investor's demand for the risky asset. Barberis and Huang (2008) provide an alternative proof for the existence of CAPM under CPT assuming normally distributed returns, and show the existence of equilibria in the case of normally distributed returns and homogeneity of preferences. We do not examine the existence or non-existence of financial market equilibria, and hence do not use the usual arbitrage arguments utilized by De Giorgi, Hens and Levy (2004), De Giorgi and Hens (2006), or De Giorgi, Hens, and Rieger (2008). Indeed, we

\footnotetext{
${ }^{4}$ See for example Allais (1953), Ellsberg (1961), Edwards (1962), Fellner (1961), Handa (1977) and Kahneman and Tversky (1979).

${ }^{5}$ For a survey of the developments in non-expected utility models of choice see Fishburn (1988) or Starmer (2000).
} 
are only interested in modeling the demand for the risky asset in a static oneperiod model. This problem has been recently solved by Jin and Zhou (2008) in a continuous-time setting, within the complete market framework of Black and Scholes. Their result is thus only valid for Log-Normally distributed risky asset prices.

Finally, one should note that the model we adopt here is based on the piecewisepower utility function, i.e. the model originally introduced to the literature by Tversky and Kahneman (1992). While this is the original formulation of Cumulative Prospect Theory, it has been repeatedly emphasized that this specific form of the value function violates loss aversion as defined by Köbberling and Wakker (2005), unless the shape parameters are identical (and the coefficient of loss aversion is greater than 1). See for example Köbberling and Wakker (2005) or De Giorgi Hens and Levy (2004) who suggest a piecewise-CARA value function based on exponential utilities. Our model is based on the piecewise-power value function and might hence violate loss aversion, at least in the sense of Köbberling and Wakker (2005). However, we shall explain why ours is still a valid conceptual framework.

The rest of this paper is organized as follows: Section 2 examines the main components of Cumulative Prospect Theory (CPT) and defines the CPT-objective function; Section 3 introduces the model and the portfolio selection problem, and derives the optimal holding in the risky asset; Section 4 analyzes the optimal holding in the risky asset, examines its relation to the Omega measure, and discusses the conceptual resemblance to Merton's CRRA Expected-Utility-maximizer; Section 5 considers a numerical example; Section 6 summarizes the results of this paper and concludes. Mathematical background and some of the proofs are presented in the Appendix.

\section{The CPT-investor}

In this section, we recall the framework of Tversky and Kahneman (1992) and explain the main components of Cumulative Prospect Theory (CPT). We precisely describe the three elements of the decision making of a CPT-investor: an investor who behaves consistently with CPT. Tversky and Kahneman (1992) consider discrete probability distributions. Similarly to Barberis and Huang (2008) and Jin and Zhou (2008), notation has been slightly modified to account for more general distributions.

CPT has three important components that makes it strongly different from 
Expected Utility Theory (EUT). First, the CPT-investor is concerned with the deviation of her final wealth from a reference level, whereas the Expected-Utility maximizing investor is interested only in the final value of her wealth. Second, the CPT-investor reacts differently towards gains and losses. Third, investors do not value random outcomes using the physical probabilities but base their decisions upon distorted probabilities. The distortion is typically such that low probabilities are overestimated.

We now formalize these three components explaining the decision of the CPTinvestor and introduce the notation that will be adopted throughout our analysis. Let $W$ denote the final wealth and $W^{\text {ref }}$ be the reference level of wealth at the end of the period. Define the deviation $D$ from the reference level by:

$$
D=W-W^{r e f}
$$

The cumulative distribution function (cdf) and the decumulative distribution function (ddf) of the random variable $D$ are respectively denoted by $F_{D}$ and by $S_{D} . D$ is the random variable that drives all decisions. We first define the value function $u$.

Definition 2.1. The value function $u$ is defined as follows ${ }^{6}$ :

$$
u(x)= \begin{cases}u^{+}(x) & \text { if } x \geqslant 0 \\ -u^{-}(-x) & \text { if } x<0\end{cases}
$$

where $u^{+}: \overline{\mathbb{R}}^{+} \rightarrow \overline{\mathbb{R}}^{+}$and $u^{-}: \overline{\mathbb{R}}^{+} \rightarrow \overline{\mathbb{R}}^{+}$satisfy ${ }^{7}$

- $u(0)=u^{+}(0)=u^{-}(0)=0$;

- $u^{+}(+\infty)=u^{-}(+\infty)=+\infty$;

- $u^{+}(x)=x^{\alpha}$, with $0<\alpha<1$ and $x \geqslant 0$;

- $u^{-}(x)=\lambda x^{\beta}$, with $\alpha \leqslant \beta<1, \lambda>1$ and $x \geqslant 0$.

Figure 1 illustrates the value function $u(x)$ for different values of $\alpha, \beta$ and $\lambda$.

Two crucial remarks follow: (i) Since x represents a deviation in wealth rather than an amount of wealth, the concept of diminishing marginal returns can no longer be seen as represented by the concavity/curvature of $u^{+}$or $u^{-}$(As noted by Davies and Satchell (2007)); and (ii) $1-\beta=-x\left(\frac{\frac{d^{2}\left(-u^{-}(-x)\right)}{d x^{2}}}{\frac{d(-u-(-x))}{d x}}\right)>0$ is not a

\footnotetext{
${ }^{6}$ We use the definition of Tversky and Kahneman (1992).

${ }^{7}$ Note that $\overline{\mathbb{R}}^{+}$denotes $\mathbb{R}^{+} \cup\{+\infty\}$.
} 


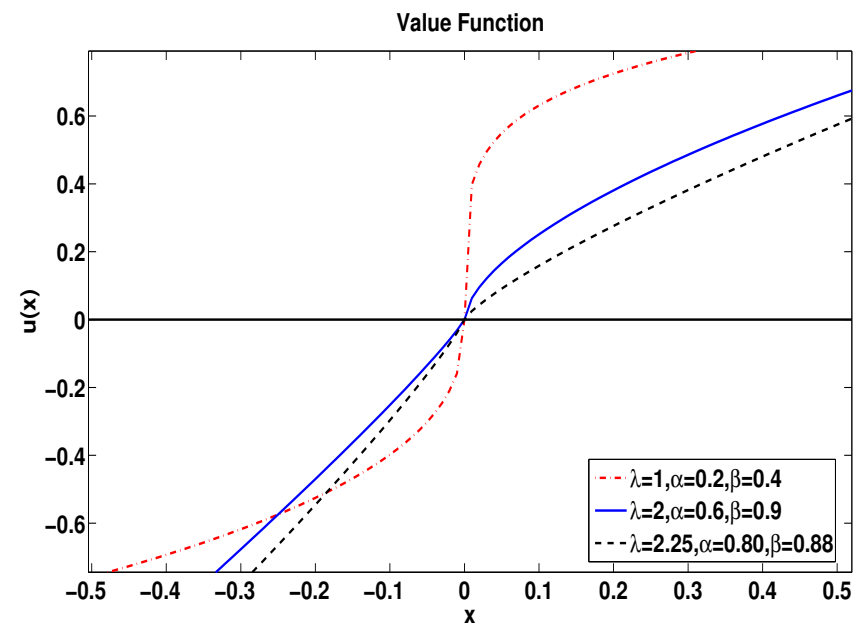

Figure 1: The value function $\mathrm{u}(\mathrm{x})$ for different values of $\alpha, \beta$ and $\lambda$.

The value function is concave over gains and is convex over losses.

measure of loss aversion in the understanding of Cumulative Prospect Theory. It is simply a measure of the affinity or disparity to utilitarian risk implied by the curvature of $u^{-}$, ceteris paribus.

The third component of the decision of a CPT-investor lies in the systematic distortion of the physical probability measure. The probability distortion process may be slightly different for losses (negative deviations $D$ ) or for gains (positive deviations $D$ ). They are defined as follows 8 .

Definition 2.2. The probability distortions (or probability weighting functions) are denoted by $T^{+}$and $T^{-}$. For a random variable $D$ with cumulative distribution function (cdf) $F_{D}$ and decumulative distribution function (ddf) $S_{D}$, we define the following two probability weighting functions (distortions) $T^{+}:[0,1] \rightarrow[0,1]$ and $T^{-}:[0,1] \rightarrow[0,1]:$

$$
\begin{aligned}
& T^{+}\left(F_{D}(x)\right)=\frac{F_{D}^{\gamma}(x)}{\left(F_{D}^{\gamma}(x)+S_{D}^{\gamma}(x)\right)^{1 / \gamma}}, \quad \text { with } 0.28<\gamma<1 \\
& T^{-}\left(F_{D}(x)\right)=\frac{F_{D}^{\delta}(x)}{\left(F_{D}^{\delta}(x)+S_{D}^{\delta}(x)\right)^{1 / \delta}}, \quad \text { with } 0.28<\delta<1 .
\end{aligned}
$$

Figure 2 illustrates the probability distortion functions within CPT.

\footnotetext{
${ }^{8}$ We use the definition of Tversky and Kahneman (1992).
} 


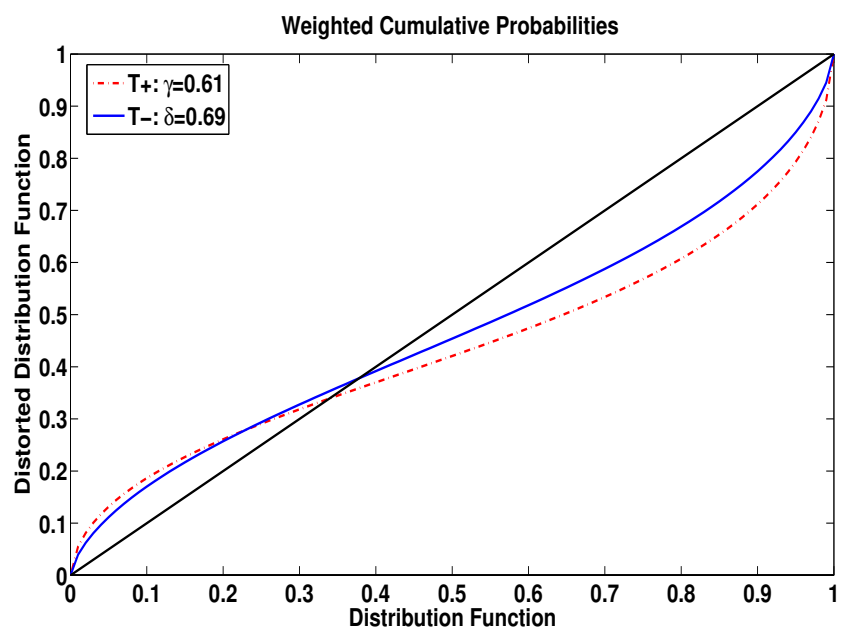

Figure 2: The distorted cumulative probabilities $T^{+}(F(x))$ and $T^{-}(F(x))$ when $\gamma=0.61$ and $\delta=0.69$ (parameters proposed by Tversky and Kahne$\operatorname{man}(1992))$.

One can easily verify that:

- The functions $u^{+}: \overline{\mathbb{R}}^{+} \rightarrow \overline{\mathbb{R}}^{+}$and $u^{-}: \overline{\mathbb{R}}^{+} \rightarrow \overline{\mathbb{R}}^{+}$are increasing, twice differentiable, invertible, positively homogeneous (with degrees $\alpha$ and $\beta$, respectively) and concave.

- The distortion functions $T^{+}:[0,1] \rightarrow[0,1]$ and $T^{-}:[0,1] \rightarrow[0,1]$ are differentiable with $T^{+}(0)=T^{-}(0)=0$ and $T^{+}(1)=T^{-}(1)=1$. Assuming $\gamma>0.28$, and $\delta>0.28$ ensures that $T^{+}$and $T^{-}$are increasing (see Barberis and Huang (2008)).

Definition 2.3. Objective function of the CPT-investor.

We define the objective function of the CPT-investor, denoted by $V^{c p t}(D)$, as:

$$
V^{c p t}(D)=\int_{0}^{+\infty} T^{+}\left(S_{D}(x)\right) d u^{+}(x)+\int_{-\infty}^{0} T^{-}\left(F_{D}(x)\right) d u^{-}(-x)
$$


$V^{c p t}(D)$ is a sum of two Choquet integrals ${ }^{9}$. It is well-defined when

$$
\alpha<2 \min (\delta, \gamma) \text { and } \beta<2 \min (\delta, \gamma)
$$

for all probability distributions. This condition is not necessary for some probability distributions, such as Log-Normal or Normal distributions. The fact that this condition ensures that both integrals are finite is proved by Barberis and Huang $(2008)^{10}$.

Proposition 2.1. The CPT-objective function $V^{\text {cpt }}(D)$ also writes as:

$$
V^{c p t}(D)=\int_{0}^{+\infty} T^{+}\left(S_{u^{+}\left(D^{+}\right)}(x)\right) d x-\int_{0}^{+\infty} T^{-}\left(S_{u^{-}\left(D^{-}\right)}(x)\right) d x,
$$

or

$$
V^{c p t}(D)=\int_{0}^{+\infty} T^{+}\left(S_{D}(x)\right) d u^{+}(x)-\int_{0}^{+\infty} T^{-}\left(F_{D}(-x)\right) d u^{-}(x),
$$

where:

$$
D^{+}=\max (D, 0), \quad D^{-}=-\min (D, 0) .
$$

The two formulations in Proposition 2.1 follow from an integration by parts and a change of variables such as Barberis and Huang (2008) do when $\alpha<\min (\delta, \gamma)$. It can also be derived under the more general assumption that $T^{-} \circ P$ and $T^{+} \circ P$ are finite. The proof is given in Appendix A.

This proposition gives two alternative formulations of $V^{c p t}(D)$. Note that Barberis and Huang work with the formulation (5). Jin and Zhou (2008) use a similar form to (6). In particular, $V^{c p t}(D)$ can be expressed as the difference between two regular integrals over $\mathbb{R}^{+}$(see formula (6)). To derive the optimal portfolio, we will often use the formulation (7).

\section{Violation of Loss Aversion}

In this paragraph, we first review how the behavioral criterion of loss aversion has been quantified in earlier work, and how our model violates the well-accepted

\footnotetext{
${ }^{9} \mathrm{~A}$ formal construction of $V^{c p t}(D)$ is given in Appendix A. $V^{c p t}(D)$ is the special case of the CPT-functional defined in Appendix A, when the variable of interest is the deviation $D$, the function $u$ is as defined in (2) and $T^{+}$and $T^{-}$are the probability distortion functions defined in (3) and (4).

${ }^{10}$ In the setting of Barberis and Huang (2008), $\alpha=\beta$ and $\delta=\gamma$, and hence the condition required is that $\alpha<2 \delta$.
} 
measure of loss aversion introduced by Köbberling and Wakker (2005). The latter is defined as

$$
L A_{k w}=\frac{\lim _{x \rightarrow 0^{-}}\left(u^{-}\right)^{\prime}(x)}{\lim _{x \rightarrow 0^{+}}\left(u^{+}\right)^{\prime}(x)} .
$$

The philosophical gist of Köbberling and Wakker (2005) can be traced back to Wakker (1994). The central task of Wakker (1994) was the search for foundations to risk attitude outside marginal utility, e.g. through a "probabilistic risk attitude" resulting from the probability distortion process within rank-dependent utility (RDU).

This trail of thought led Köbberling and Wakker (2005) to apply to Cumulative Prospect Theory (CPT) the same philosophical insight that Wakker (1994) applied to RDU, hence formulating the now standard index of loss aversion, where utility is prior to risk and loss aversion. Risk attitude is then decomposed into three parts: (i) the basic utility, (ii) probability weighting, and (iii) loss aversion as a behavioral concept, measured through the index of loss aversion that the authors introduce. However, as pointed out by numerous authors ${ }^{11}$, no single unique measure of the behavioral criterion of loss aversion exists in the literature, and the many different alternatives introduced have their advantages and their flaws. Furthermore, Schmidt and Zank (2005) argued that the idea that loss aversion is reflected in the value function being steeper for losses than for gains is inherited from the Original Prospect Theory of Kahneman and Tversky (1979) (OPT) - and clearly from Expected Utility Theory (EUT) where the curvature of the utility captures all risk attitudes, and is not applicable under CPT (Tversky and Kahneman (1992)), because it overlooks rank dependence. Consequently, a measure of loss aversion that does not account for the probability weighting process might lead to contradictions with the behavioral criterion of loss aversion, as the authors have shown.

This led Schmidt and Zank (2005) to introduce a quantitative definition of loss aversion in terms of both the utility and probability weighting ${ }^{12}$. Along the same path, Zank (2009) argues that loss aversion, being a behavioral phenomenon, is a property of choice behavior, and hence he defines a probabilistic index of loss aversion to complement the utilitarian index of loss aversion. In sum, there exists no unique universally agreed-upon measure of loss aversion as of yet, and Köbberling and Wakker (2005)'s index of loss aversion is only one of the proposed measures. Now, given the observation of loss aversion as a behavioral phenomenon, this raises the question of whether one can empirically measure loss aversion without any

\footnotetext{
${ }^{11}$ For instance, Neilson (2002), Köbberling and Wakker (2005), Abdellaoui, Bleichrodt and Paraschiv (2007), and Zank (2009).

${ }^{12}$ See also Davies and Satchell (2007).
} 
prior postulated parametrization. This has been done by Abdellaoui, Bleichrodt and Paraschiv (2007). The authors found empirical support for the existence of loss aversion as a behavioral criterion without any parametric assumption on the value function. Their results also suggest that a power-value function is best suited.

Here, we prefer not to partake in the debate of whether a measure of loss aversion should be derived from the utility function, from the probability distortion functions, or from both. Instead, we will simply define loss aversion as the behavioral phenomenon that "losses loom larger than gains" (Kahneman and Tversky (1979)), i.e. that losses matter more than gains, for a given reference point, and for a given deviation from the reference point ${ }^{13}$. When the value-function is a piecewise-power value function with different shape parameters, unless $\alpha=\beta$, our model violates loss aversion as shown hereafter. Precisely, if $a>0$ is a fixed real number, then

$$
\begin{aligned}
V^{+}(a) & =\int_{0}^{+\infty} T^{+}\left(\operatorname{Pr}\left[u^{+}(a)>t\right]\right) d t=\int_{0}^{+\infty} T^{+}(1) \cdot \mathbb{1}_{u^{+}(a)>t} d t \\
& =\int_{0}^{u^{+}(a)} 1 d t=u^{+}(a) .
\end{aligned}
$$

Similarly, $V^{-}(a)=u^{-}(a)$. Hence for any $x>0$, we have $V^{c p t}(x)=u^{+}(x)=x^{\alpha}$ and $\left|V^{c p t}(-x)\right|=\left|-u^{-}(x)\right|=\lambda x^{\beta}$. Consequently, for all $x>0$, loss aversion as a behavioral criterion holds when $V^{c p t}(x)<\left|V^{c p t}(-x)\right|$, that is when $x^{\alpha}<\lambda x^{\beta}$, i.e.

$$
\lambda>x^{\alpha-\beta} .
$$

Obviously, when $\alpha<\beta$, this will not be satisfied for small deviations from the reference level, when

$$
x<\varepsilon:=\lambda^{\frac{1}{\alpha-\beta}} .
$$

As a conclusion, our model violates loss aversion when $\alpha<\beta$, when deviations from the reference level are small, and when "loss aversion" is defined by a criterion that does not take into account the distortion of the probabilities, but depends only on the utility functions. But some recent experimental studies show that individuals decisions sometimes violate loss aversion ${ }^{14}$. It is thus still interesting to study the model when $\alpha<\beta$.

\footnotetext{
${ }^{13}$ See also Wakker and Tversky (1993) and Starmer (2000).

${ }^{14}$ See for instance Baucells and Heukamp (2006).
} 


\section{A one-risky-one-risk-free asset market}

In this section, we derive the optimal portfolio choice for the CPT-investor in a one-period economy. Notation and assumptions are consistent with the standard framework presented in Chapter 4 of Gollier (2001), for instance, in the context of Expected Utility Theory.

\subsection{Setting}

Consider a one-period static portfolio choice problem. The financial market consists of one risk-free asset, with return $r$ over the period, and one risky asset with stochastic return $\widetilde{x}$ over the period. Let $W_{0}$ denote the investor's initial wealth. An amount $\zeta$ is invested in the risky asset and the remaining wealth, $W_{0}-\zeta$, is invested in the risk-free asset. We assume that short-selling is forbidden, i.e. $\zeta \geqslant 0$. If the investor can borrow to invest in the risky asset, then $\zeta$ may exceed $W_{0}$. We will discuss the case where both short-selling and borrowing are not allowed, and the case where only borrowing is allowed. The individual's wealth at the end of the period is given by:

$$
W=\left(W_{0}-\zeta\right)(1+r)+\zeta(1+\widetilde{x})=W_{0}(1+r)+\zeta(\widetilde{x}-r)
$$

Now, define $\widetilde{y}$, the excess return on the risky asset over the risk-free rate as

$$
\widetilde{y}=\widetilde{x}-r
$$

We assume that $\widetilde{y}$ can take both positive values and negative values with positive probabilities. Define the reference level of wealth at the end of the period, $W^{\text {ref }}$, as

$$
W^{r e f}=W_{0}(1+r)
$$

$W^{r e f}$ is the amount the individual would have received at the end of the period had he invested all of his initial wealth $W_{0}$ in the risk-free asset (e.g. in a bank account). Then the individual's wealth at the end of the period is given by:

$$
W=W^{r e f}+\zeta \widetilde{y}
$$

The deviation from the reference level at the end of the period then satisfies:

$$
D(\zeta)=W-W^{r e f}=\zeta \widetilde{y} .
$$




\subsection{Optimal Portfolio Choice}

Consider a CPT-investor as defined in section 2. In this setting, the objective function of the CPT-investor, $V^{c p t}$, writes as follows:

$$
V^{c p t}(D(\zeta))=\zeta^{\alpha} \int_{0}^{+\infty} T^{+}\left[S_{\widetilde{y}}(y)\right] d u^{+}(y)-\zeta^{\beta} \int_{0}^{+\infty} T^{-}\left[F_{\widetilde{y}}(-y)\right] d u^{-}(y)
$$

ation

Equation (9) is derived in Appendix B.1. The portfolio choice problem consists of finding the optimal amounts to allocate to the risky asset and to the risk-free asset. The allocation problem becomes:

$$
\max _{\zeta \geqslant 0} V^{c p t}(D(\zeta))
$$

and using equation (9), it can be rewritten as follows:

$$
\max _{\zeta \geqslant 0}\left(\mathbb{G}(\widetilde{y}) \zeta^{\alpha}-\mathbb{L}(\widetilde{y}) \zeta^{\beta}\right)
$$

where

$$
\left\{\begin{array}{l}
\mathbb{G}(\widetilde{y})=\int_{0}^{+\infty} T^{+}\left[S_{\widetilde{y}}(y)\right] d u^{+}(y) \\
\mathbb{L}(\widetilde{y})=\int_{0}^{+\infty} T^{-}\left[F_{\widetilde{y}}(-y)\right] d u^{-}(y)
\end{array}\right.
$$

Note that $\mathbb{G}(\widetilde{y})$ and $\mathbb{L}(\widetilde{y})$ are positive quantities that do not depend on the portfolio allocation $\zeta$. Their difference is equal to:

$$
\mathbb{G}(\widetilde{y})-\mathbb{L}(\widetilde{y})=V^{c p t}(\widetilde{y}) .
$$

Let us denote by $\Omega(\widetilde{y})$ the ratio of $\mathbb{G}(\widetilde{y})$ to $\mathbb{L}(\widetilde{y})$. It will play a key role in determining the optimal holding in the risky asset of the CPT-investor. Throughout the paper, we refer to $\Omega(\widetilde{y})$ as the CPT-ratio.

$$
\Omega(\widetilde{y})=\frac{\mathbb{G}(\widetilde{y})}{\mathbb{L}(\widetilde{y})}=\frac{\int_{0}^{+\infty} T^{+}\left[S_{\widetilde{y}}(y)\right] d u^{+}(y)}{\int_{0}^{+\infty} T^{-}\left[F_{\widetilde{y}}(-y)\right] d u^{-}(y)} .
$$

After straightforward computations, $\Omega(\widetilde{y})$ can also be written as:

$$
\Omega(\widetilde{y})=\frac{\int_{0}^{+\infty} T^{+}\left[S_{u^{+}\left(\widetilde{y}^{+}\right)}(y)\right] d y}{\int_{0}^{+\infty} T^{-}\left[S_{u^{-}\left(\widetilde{y}^{-}\right)}(y)\right] d y}
$$

where $\widetilde{y}=\widetilde{y}^{+}-\widetilde{y}^{-}$. This last expression shows that $\mathbb{G}(\widetilde{y})$ depends only on the distribution of gains while $\mathbb{L}(\widetilde{y})$ depends only on that of losses. 


\subsection{Optimal Portfolio}

Here we solve for the optimal portfolio of the CPT-investor (problem (10)). We first consider the situation where only borrowing is allowed (i.e. $\zeta \geqslant 0)$, and we then consider the case where short-selling and borrowing constraints are imposed (i.e. $\left.\zeta \in\left[0, W_{0}\right]\right)$.

Theorem 3.1. Given $0<\alpha \leqslant \beta<\min \{1,2 \min (\delta, \gamma)\}$, denote by $\zeta_{*} \in \overline{\mathbb{R}}^{+}$ the optimal holding in the risky asset of the CPT-investor. $\zeta_{*}$ solves the following maximization problem:

$$
\max _{\zeta \in \overline{\mathbb{R}}^{+}}\left(\mathbb{G}(\widetilde{y}) \zeta^{\alpha}-\mathbb{L}(\widetilde{y}) \zeta^{\beta}\right)
$$

Furthremore:

- If $\alpha=\beta$, then there are three cases to consider:

- if $V^{c p t}(\widetilde{y})=0$, any holding in the risky asset is optimal. The objective function is constant and equal to 0.

- if $V^{c p t}(\widetilde{y})>0$, it is optimal to borrow an infinite amount to invest in the risky asset. The objective function is then equal to $+\infty$.

- if $V^{c p t}(\widetilde{y})<0$, then the optimal amount to invest in the risky asset is $\zeta_{*}=0$.

- If $\alpha<\beta$, then the optimal holding in the risky asset $\zeta_{*}$ is given by:

$$
\zeta_{*}=\left(\frac{\alpha}{\beta}\right)^{\frac{1}{\beta-\alpha}} \Omega(\widetilde{y})^{\frac{1}{\beta-\alpha}}
$$

A few comments follow. The optimal amount to invest in the risky asset does not depend on $W_{0}$. It depends only on the characteristics of the distribution of the excess return on the risky asset over the risk-free rate. At first sight, this result might seem surprising. However, we are only interested in the deviation from the final wealth. The reference point depends on the initial wealth, but the deviation from the reference point (given by (8)) does not depend on $W_{0}$. The problem of optimal portfolio choice stated in (10) does not depend on $W_{0}$. Nevertheless two investors with very different initial wealth may have different behaviors and therefore different parameters $\alpha$ and $\beta$. 
Proof. If $\alpha=\beta$, then we can write $V^{c p t}(D)=\zeta^{\alpha} V^{c p t}(\widetilde{y})$ and the three cases are straightforward. When $\alpha \neq \beta$, the first-order condition is given by:

$$
\frac{d\left(\mathbb{G}(\widetilde{y}) \zeta^{\alpha}-\mathbb{L}(\widetilde{y}) \zeta^{\beta}\right)}{d \zeta}=0
$$

which yields the only root $\zeta_{*}$ :

$$
\zeta_{*}:=\left(\frac{\beta \mathbb{L}(\widetilde{y})}{\alpha \mathbb{G}(\widetilde{y})}\right)^{\frac{1}{\alpha-\beta}}=\left(\frac{\alpha}{\beta}\right)^{\frac{1}{\beta-\alpha}} \Omega(\widetilde{y})^{\frac{1}{\beta-\alpha}}
$$

The second-order condition is given by verifying:

$$
\frac{d^{2}\left(\mathbb{G}(\widetilde{y}) \zeta^{\alpha}-\mathbb{L}(\widetilde{y}) \zeta^{\beta}\right)}{d \zeta^{2}}<0
$$

or equivalently,

$$
(\alpha-1) \zeta^{\alpha-2}<\zeta_{*}^{\alpha-\beta}(\beta-1) \zeta^{\beta-2}
$$

when $\zeta=\zeta_{*}$. Thus, one obtains $(\alpha-1) \zeta_{*}^{\alpha-2}<(\beta-1) \zeta_{*}^{\alpha-2}$. Hence, the second-order condition is:

$$
\alpha<\beta
$$

Which is given by hypothesis. This hence gives the optimal solution $\zeta_{*}$.

Theorem 3.2. Given $0<\alpha \leqslant \beta<\min \{1,2 \min (\delta, \gamma)\}$, suppose that shortselling is prohibited and investors are not allowed to borrow in order to invest in the risky asset. Denote by $\zeta_{*} \in\left[0, W_{0}\right]$ the optimal holding in the risky asset of the CPT-investor. $\zeta_{*}$ solves the following maximization problem:

$$
\max _{\zeta \in\left[0, W_{0}\right]}\left(\mathbb{G}(\widetilde{y}) \zeta^{\alpha}-\mathbb{L}(\widetilde{y}) \zeta^{\beta}\right)
$$

Furthermore:

- If $\alpha=\beta$, then there are three cases to consider:

- if $V^{c p t}(\widetilde{y})=0$, any holding in the risky asset is optimal. The objective function is constant and equal to 0.

- if $V^{c p t}(\widetilde{y})>0$, then it is optimal to invest $W_{0}$ in the risky asset.

- if $V^{c p t}(\widetilde{y})<0$, then the optimal amount to invest in the risky asset is $\zeta_{*}=0$. 
- If $\alpha<\beta$, then the optimal holding in the risky asset $\zeta_{*}$ is given by:

$$
\zeta_{*}=\min \left(\left(\frac{\alpha}{\beta}\right)^{\frac{1}{\beta-\alpha}} \Omega(\widetilde{y})^{\frac{1}{\beta-\alpha}}, W_{0}\right) .
$$

Proof. The result of Theorem 3.2 is a direct consequence of Theorem 3.1. In the presence of borrowing constraints, the optimal holding is bounded by $W_{0}$ when it is greater than $W_{0}$.

Theorem 3.2 shows that, given $0<\alpha<\beta<\min \{1,2 \min (\delta, \gamma)\}$, the optimal holding is not trivial if and only if $\Omega(\widetilde{y})$ satisfies:

$$
\Omega(\widetilde{y})<\Omega_{\max }:=\left(\frac{\beta}{\alpha}\right) W_{0}^{\beta-\alpha}
$$

If $\Omega(\widetilde{y})$ exceeds $\Omega_{\max }$, the optimal holding is trivial and the total wealth is invested in the risky asset. Note that the problem of optimal portfolio choice (17) now depends on $W_{0}$. The optimal amount to invest in the risky asset has an upper bound $W_{0}$ which implies an upper bound on how the risky asset performs, measured by the Omega of its excess return, $\Omega(\widetilde{y})$.

$\Omega(\widetilde{y})$ is a positive quantity that depends only on the distribution of $\widetilde{y}$, the excess return of the risky asset over the risk-free rate. In the next section, we will provide more economic interpretation of the CPT-ratio $\Omega(\widetilde{y})$ and see it as a performance measure.

\section{Optimal portfolio of the CPT-investor}

In this section, we study the optimal investment in the risky asset when it is not trivial. Hence we suppose $\alpha<\beta$ and $\Omega(\widetilde{y})$ is bounded by $\Omega_{\max }$ defined in (19). Under these assumptions, the optimal holding of the CPT-investor in the risky asset is given by:

$$
\zeta_{*}=\Omega(\widetilde{y})^{\frac{1}{\beta-\alpha}}\left(\frac{\alpha}{\beta}\right)^{\frac{1}{\beta-\alpha}}
$$

where $\Omega(\widetilde{y})$ is given by equations (12) or (13). The study of the optimal holding is closely linked to the study of the CPT-ratio. In particular, 
Proposition 4.1. The higher the CPT-ratio the higher the optimal allocation in the risky asset. Formally:

$$
\Omega\left(\widetilde{y}_{1}\right) \geqslant \Omega\left(\widetilde{y}_{2}\right) \Rightarrow \zeta_{*}\left(\widetilde{y}_{1}\right) \geqslant \zeta_{*}\left(\widetilde{y}_{2}\right)
$$

We first interpret $\Omega$ as a performance measure and then highlight the similarity between the optimal holding of the CPT-investor and Merton (1969)'s result for the CRRA expected-utility maximizing investor. Note that all results in this section are independent of the distribution of the risky asset, but clearly depend on the specific choice of reference point made in this paper.

\section{1 $\Omega(\widetilde{y})$ as a performance measure}

The optimal holding in the risky asset of the CPT-investor depends on the CPT-ratio $\Omega(\widetilde{y})$. This ratio quantifies the upside potential of the risky asset (measured by $\mathbb{G}(\widetilde{y})$ ) relative to the downside potential of that risky asset (measured by $\mathbb{L}(\widetilde{y})$ ). Hence it can be interpreted as a performance measurement ratio and as a generalized form of the Omega measure ${ }^{15}$ (see Keating and Shadwick (2002) or Cascon, Keating and Shadwick (2003)), or as a generalized form of the Gain-Loss Ratio of Bernardo and Ledoit (2000). We define below these two measures and see how they are related to the CPT-investor's optimal holding in the risky asset.

Definition 4.1. The Omega measure (Keating and Shadwick (2002))

For a given return random variable $\widetilde{x}$ with cdf $F$ and support $(a, b)$ on the real line, the Omega measure at threshold level $L$ is given by:

$$
\bar{\Omega}_{F}(L)=\frac{\int_{L}^{b}[1-F(x)] d x}{\int_{a}^{L} F(x) d x}=\frac{E_{\mathbb{P}}\left[(\widetilde{x}-L)^{+}\right]}{E_{\mathbb{P}}\left[(L-\widetilde{x})^{+}\right]}>0
$$

where expectations are taken under the physical probability measure $P$.

$L$ is seen as the benchmark level of return such that any realization of the random return $\widetilde{x}$ beyond $L$ is interpreted as a gain (reward), and any realization below $L$ is a loss. The Omega measure is hence a ratio of a probabilityweighted sum of gains (values above the threshold) to a probability-weighted

\footnotetext{
${ }^{15}$ Note that De Giorgi, Hens and Mayer (2006) have already pointed out the implications of Omega performance measures and similar quantities in CPT.
} 
sum of losses (values below the threshold). For a given threshold $L$, the higher the value of $\bar{\Omega}$, the more desirable the investment.

Definition 4.2. The Gain-Loss Ratio (Bernardo and Ledoit (2000)) For any excess return random variable $\widetilde{y}$, define $\widetilde{y}^{+}$and $\widetilde{y}^{-}$to be its positive and negative parts, respectively, so that $\widetilde{y}=\widetilde{y}^{+}-\widetilde{y}^{-}$. Then, the Gain-Loss ratio for $\widetilde{y}$ is defined as:

$$
G L_{\widetilde{y}}=\frac{E^{*}\left[\widetilde{y}^{+}\right]}{E^{*}\left[\widetilde{y}^{-}\right]}
$$

where $E^{*}[$.$] denotes the expectation with respect to a risk-adjusted probability$ measure $P^{*}$ reflecting some benchmark pricing kernel, such as the marginal rate of substitution between initial and end-of-period states.

The CPT-ratio $\Omega(\widetilde{y})$ is a generalization of the omega measure as well as of the Gain-loss ratio. It is a ratio of gains over losses where the physical measure is distorted and expectations of the S-shaped preferences replace the standard expectation. This can be seen from (13) for instance. Furthermore if we define the random variable $\widetilde{y}$ to be the excess return over the benchmark level $L=0$, then any positive realization of $\widetilde{y}$ is seen as a gain, and any negative realization of $\widetilde{y}$ is seen as a loss. In some sense $\Omega(\widetilde{y})$ is an omega measure evaluated under the subjective distorted measure rather than the physical measure, and where the investor has S-shaped preferences instead of being risk-neutral.

The Omega measure or the Gain-Loss ratio are used to evaluate the asymmetry of a distribution. They have been introduced to generalize symmetric measures such as the Sharpe ratio. Fatter tails for the gains imply a higher CPT-ratio. In some sense the CPT-ratio assesses the quality of the risky asset. Similarly to the Omega measure or the Gain-Loss ratio, the CPTratio accounts for the whole distribution and its moments. In particular, the CPT-ratio reflects the skewness and asymmetry of a distribution, as will be seen in section 5. As a consequence of Proposition 4.1, the CPT-investor is sensitive to asymmetric distributions and invests more wealth in risky assets with fat tails of gains.

\subsection{Merton's investor and the CPT-investor}

In the classical Merton portfolio choice problem where returns are assumed to be normally distributed (Merton (1969)), and the investor has a 
CRRA utility ${ }^{16}$, the optimal amount to be invested in the risky asset is given by:

$$
\zeta_{*}^{M}=\left(\frac{\mu-r}{\sigma^{2}}\right) \frac{1}{1-a} W_{0}
$$

where $1-a$ is the (constant) relative risk aversion with $0<a<1, \mu$ is the expected return on the normally distributed risky asset, and $\sigma^{2}$ is the variance of the return on the risky asset.

This optimal portfolio has two components: (i) $\frac{\mu-r}{\sigma^{2}}$ is a measure of the "performance" of the investment in the risky asset (defined as the Sharpe ratio divided by the volatility parameter $\sigma$ ), and (ii) $\frac{1}{1-a}$ is a measure of the curvature of the CRRA investor's utility function.

Hence, in essence, the optimal amount invested by the CPT-investor in the risky asset shares similarities with Merton's result for the CRRA expected-utility maximizer. The optimal holding $\zeta_{*}$ (given by (20)) of the CPT-investor has two components that play similar roles to the ones played by the components of the optimal holding of Merton's CRRA investor: (i) $\Omega(\widetilde{y})$ can be seen as a risk-reward measure associated with the risky excess return $\widetilde{y}$, and (ii) the parameters $\alpha$ and $\beta$ are related to the curvature of the value function on the positive and negative domains, respectively ${ }^{17}$.

\subsection{Properties of the optimal allocation}

In this section we examine some properties of the CPT-Ratio, $\Omega(\widetilde{y})$, given in equation (12) or (13) and thus of the optimal holding in the risky asset:

$$
\zeta_{*}(\widetilde{y})=\left[\Omega(\widetilde{y})\left(\frac{\alpha}{\beta}\right)\right]^{\frac{1}{\beta-\alpha}}
$$

Note that the optimal holding is neither an increasing nor a decreasing function of $\alpha$ or $\beta$ (as measures of curvature of the value function), which can also be seen in the numerical study in section 5 (see Figures 5 and 6). This is already drastically different from the Expected Utility framework where

\footnotetext{
${ }^{16}$ Note that the functions $u^{+}$and $u^{-}$in the context of CPT both exhibit CRRA.

${ }^{17}$ It should be noted that the risk-reward measure considered here in the context of CPT is preference-dependent, whereas $\frac{\mu-r}{\sigma^{2}}$ is not. However, both $\zeta_{*}$ and $\zeta_{*}^{M}$ are obviously preference-dependent.
} 
higher risk aversion (curvature of the utility function) usually implies a lower investment in the risky asset.

Proposition 4.2. Homogeneity.

Suppose $\alpha<\beta$ and $\Omega(\widetilde{y})<\Omega_{\max }$. Given $m>0$, the optimal holding in the risky asset is (positively) homogeneous of degree -1 , that is:

$$
\zeta_{*}(m \widetilde{y})=\frac{1}{m} \zeta_{*}(\widetilde{y})
$$

and the CPT-Ratio $\Omega(\widetilde{y})$ is positively homogeneous of degree $\alpha-\beta$,

$$
\Omega(m \widetilde{y})=m^{\alpha-\beta} \Omega(\widetilde{y})
$$

The proof is given in the appendix.

Equation (23) asserts that if the risk is proportionally increased by a factor $m>1$, then the optimal holding in the risky asset is proportionally reduced by the same factor. Note that equation (24) holds even for $\alpha \geqslant \beta$. In particular, in the case where the CPT-investor has a piecewise-power value function such that loss aversion holds, i.e. $\alpha=\beta$ and $\lambda>1$, we have that $\Omega(m \widetilde{y})=\Omega(\widetilde{y})$ for all $m>0$, which way seem surprising. In effect, this implies that even if the risk is proportionally increased by a factor $m>1$, its subjective, preference-based evaluation (or performance measurement) by the loss-averse CPT-investor (given by $\Omega(\widetilde{y})$ ) remains unchanged. Might a loss-averse CPT-investor be "myopic" indeed?

A fundamental property of the CPT-ratio is that it preserves first-order stochastic dominance. This result is not surprising since the CPT-ratio is closely related to CPT preferences ${ }^{18}$.

Proposition 4.3. The CPT-ratio preserves first-order stochastic dominance. Consider two real-valued random prospects $\widetilde{y}_{1}$ and $\widetilde{y}_{2}$. If $\widetilde{y}_{1}$ first-stochastically dominates $\widetilde{y}_{2}$ (we write $\widetilde{y}_{1} \succ_{\text {fsd }} \widetilde{y}_{2}$ ), then its CPT ratio is higher. Formally,

$$
\widetilde{y}_{1} \succ_{f s d} \widetilde{y}_{2} \Rightarrow \Omega\left(\widetilde{y}_{1}\right) \geqslant \Omega\left(\widetilde{y}_{2}\right)
$$

\footnotetext{
${ }^{18}$ Tversky and Kahneman (1992) point out that CPT preferences satisfy first-order stochastic dominance. Barberis and Huang (2008) give an alternative proof of this property. Propositions 4.3 and 4.4 are consistent with these previous findings. Wang and Young (1998) also propose a proof of this result.
} 
Proof.

$$
\widetilde{y}_{1} \succ_{f s d} \widetilde{y}_{2} \Leftrightarrow \forall x \in \mathbb{R}, F_{\widetilde{y}_{1}}(x) \leqslant F_{\widetilde{y}_{2}}(x)
$$

From (11), recall that $\mathbb{L}(\widetilde{y})=\int_{0}^{+\infty} T^{-}\left[F_{\widetilde{y}}(-y)\right] d u^{-}(y)$. Since $T^{-}$is increasing,

$$
\mathbb{L}\left(\widetilde{y}_{1}\right) \leqslant \mathbb{L}\left(\widetilde{y}_{2}\right)
$$

The form of $\mathbb{G}(\widetilde{y})$ given by (11) and the fact that $T^{+}$is increasing yields:

$$
\mathbb{G}\left(\widetilde{y}_{1}\right) \geqslant \mathbb{G}\left(\widetilde{y}_{2}\right)
$$

Since $\mathbb{L}\left(\widetilde{y}_{1}\right)$ and $\mathbb{L}\left(\widetilde{y}_{1}\right)$ are positive quantities, their ratio $\Omega$ satisfies:

$$
\Omega\left(\widetilde{y}_{1}\right) \geqslant \Omega\left(\widetilde{y}_{2}\right)
$$

This completes the proof of Proposition 4.3.

A higher $\Omega(\widetilde{y})$ means a more attractive asset for the CPT-investor, and a higher optimal holding in the risky asset (see Proposition 4.1).

Consider two different distributions for the excess return of the risky asset, say two real-valued random prospects $\widetilde{y}_{1}$ and $\widetilde{y}_{2}$. We now define $D_{1}=$ $D\left(\widetilde{y}_{1}\right)=\zeta \widetilde{y}_{1}$ and $D_{2}=D\left(\widetilde{y}_{2}\right)=\zeta \widetilde{y}_{2}$, the respective deviations from the reference level $W^{\text {ref }}$.

Proposition 4.4. If $\widetilde{y}_{1}$ first-stochastically dominates $\widetilde{y}_{2}$ then for the same amount of wealth $\zeta$ invested in the risky asset, the CPT-investor prefers to invest in $\widetilde{y}_{1}$.

$$
\widetilde{y}_{1} \succ_{f s d} \widetilde{y}_{2} \Rightarrow V^{c p t}\left(D_{1}\right) \geqslant V^{c p t}\left(D_{2}\right)
$$

Proof. This is an immediate consequence of the inequalities (25) and (26) and of the expression of $V^{c p t}(D)$ given by:

$$
V^{c p t}(D(\widetilde{y}))=\mathbb{G}(\widetilde{y}) \zeta^{\alpha}-\mathbb{L}(\widetilde{y}) \zeta^{\beta}
$$

\section{Remark 4.1.}

In general, this result fails to hold for second-order stochastic dominance. Assume for instance that $\widetilde{y}_{2}$ can be obtained by modifying $\widetilde{y}_{1}$ by a meanpreserving spread. Thus $\widetilde{y}_{1}$ second-stochastically dominates $\widetilde{y}_{2}$. The effect 
of a mean-preserving spread is two-fold: It fattens the right tail of the distribution but it also fattens the left tail of the distribution. A fat tail for gains is in general an attractive feature whereas to fatten the left tail could be either attractive or not. Consequently, whether or not the effect of losses can compensate for the effect of gains will depend on the specific values of the parameters. Barberis and Huang (2008) show that CPT-investors are averse to mean-preserving spreads when $\widetilde{y}_{1}$ and $\widetilde{y}_{2}$ are both symmetrically distributed. They argue that the CPT-investor is loss-averse and therefore more sensitive to changes in the left tail than in the right tail. However, the risk-seeking behavior of the CPT-investor against losses could also imply the opposite conclusion. The result is thus ambiguous and a general conclusion cannot be reached at this stage.

\subsection{Background Risk}

We now examine how a small change in the distribution of the excess return $\widetilde{y}$ will influence the optimal holding in the risky asset. Denote by $\widetilde{z}$ an additive modification of the excess return. The deviation from the reference level is given by

$$
D(\zeta)=\zeta(\widetilde{y}+\widetilde{z})
$$

Then, the optimal holding is given by:

$$
\zeta_{*}(\widetilde{y}+\widetilde{z})=\left[\frac{\mathbb{G}(\widetilde{y}+\widetilde{z})}{\mathbb{L}(\widetilde{y}+\widetilde{z})}\left(\frac{\alpha}{\beta}\right)\right]^{\frac{1}{\beta-\alpha}}
$$

$\widetilde{z}$ can be interpreted as a particular case of background risk. In the insurance literature, Gollier (1996) examines the optimal insurance contract when the distribution of losses is not perfectly known. In the case of optimal portfolio choice in the EUT framework, some results can be found in Eeckhoudt, Gollier and Schlesinger (2005). The following proposition is for a very specific case of background risk, namely when the latter is comonotonic with the risky asset.

Proposition 4.5. Comonotonic Background Risk

Suppose that the background risk $\widetilde{z}$ is comonotonic with $\widetilde{y}^{19}$ and that all

\footnotetext{
${ }^{19}$ That is, there exists a risk $\widetilde{x}$ and non-decreasing real-valued functions $f$ and $g$ such that $\widetilde{y}=f(\widetilde{x})$ and $\widetilde{z}=g(\widetilde{x})$.
} 
previous assumptions about the parameters $\alpha, \beta, \gamma$ and $\delta$ hold. Then the numerator and denominator of the CPT-ratio are additive and the optimal holding can be written as follows:

$$
\zeta_{*}(\widetilde{y}+\widetilde{z})=\left[\frac{\mathbb{G}(\widetilde{y})+\mathbb{G}(\widetilde{z})}{\mathbb{L}(\widetilde{y})+\mathbb{L}(\widetilde{z})}\left(\frac{\alpha}{\beta}\right)\right]^{\frac{1}{\beta-\alpha}}
$$

Proof. The result follows immediately from the comonotonic additivity of the Choquet integral for monotone capacities (Denneberg (1994)).

The consequence of this proposition is that the CPT-ratio, and thus the optimal holding in the risky asset, can either increase or decrease in the presence of an additive comonotonic background risk. If the additional risk $\widetilde{z}$ affects only the gains $(\mathbb{L}(\widetilde{z})=0)$, then it will increase the optimal holding. On the other hand, if it affects only the losses $(\mathbb{G}(\widetilde{z})=0)$, then it will decrease the optimal holding in the risky asset. This is intuitive: the presence of a comonotonic risk affecting only gains will increase gains without changing losses and thus makes the risky asset more attractive. If it increases losses with no effect on gains, then it will clearly be a worse investment.

Proposition 4.6. First-Order-Stochastic-Dominant Background Risk Consider the risky prospect $\widetilde{y}$ and suppose that there exists a background risk $\widetilde{z}$ such that $\widetilde{y}+\widetilde{z}$ first-stochastically dominates $\widetilde{y}$. Then $\Omega(\widetilde{y}+\widetilde{z}) \geqslant \Omega(\widetilde{y})$, and hence $\zeta_{*}(\widetilde{y}+\widetilde{z}) \geqslant \zeta_{*}(\widetilde{y})$, when $\alpha<\beta$.

Proof. The proof is immediate based on proposition 4.3. Indeed, for any realvalued background risk $\widetilde{z}$, if $\widetilde{y}+\widetilde{z}$ first-stochastically dominates $\widetilde{y}$, then by proposition 4.3, $\Omega(\widetilde{y}+\widetilde{z}) \geqslant \Omega(\widetilde{y})$. Therefore, when $\alpha<\beta, \widetilde{y}+\widetilde{z}$ dominates $\widetilde{y}$ in the sense of first-order stochastic dominance implies that $\zeta_{*}(\widetilde{y}+\widetilde{z}) \geqslant$ $\zeta_{*}(\widetilde{y})$.

This result shows that the existence of a background risk $\widetilde{z}$, such that $\widetilde{y}+\widetilde{z}$ dominates $\widetilde{y}$ in the sense of first-order stochastic dominance, increases the demand for the risky asset, when this demand is defined and finite. Note that this is not a zero-mean background risk such as defined by Gollier (2001). More general cases are left for future research. 


\section{Example: Skew-Normal Distribution}

Since it seems difficult to obtain more information on the optimal holding in the risky asset in the general case, we now study a specific distribution for the risky return. We assume that $\widetilde{y}$, the excess return on the risky asset over the risk-free rate, has a univariate Skew-Normal distribution.

\subsection{The Univariate Skew-Normal Distribution}

Here we briefly recall the definition of a Skew-Normal distribution and some of its properties. For a formal treatment see Genton (2004). If X is a skew-normal random variable with skewness parameter $\chi$, written as $X \sim S N(\chi)$, then its pdf $f_{X}$ is given by:

$$
f_{X}(y ; \chi)=2 \phi(y) \Phi(\chi y)
$$

For all $y \in \mathbb{R}$, where $\phi$ and $\Phi$ are respectively the pdf and cdf of the standard normal distribution.

Define $Y=l+s X$, where $X \sim S N(\chi), l \in \mathbb{R}$ is a location parameter and $s>0$ is a scale parameter. The mean, variance and skewness of $Y$ are respectively given by:

$$
\begin{gathered}
E[Y]=l+s \sqrt{\frac{2}{\pi}} \frac{\chi}{\sqrt{1+\chi^{2}}}, \\
\operatorname{Var}[Y]=s^{2}\left[1-\frac{2}{\pi}\left(\frac{\chi^{2}}{1+\chi^{2}}\right)\right]
\end{gathered}
$$

and

$$
S k[X]=\frac{s^{3}}{2}(4-\pi) \operatorname{sign}(\chi)\left[\frac{\chi^{2}}{\frac{\pi}{2}+\left(\frac{\pi}{2}-1\right) \chi^{2}}\right]^{\frac{3}{2}}
$$

Figure 3 illustrates the role of the parameter $\chi$. To make the comparison possible, the location and scale parameters are respectively chosen such that the first two moments are constant (that is $E[X]$ and $\operatorname{Var}[X]$ are held constant). The sign of the parameter $\chi$ gives the sign of the skewness. The larger $|\chi|$, the more skewed the distribution. 


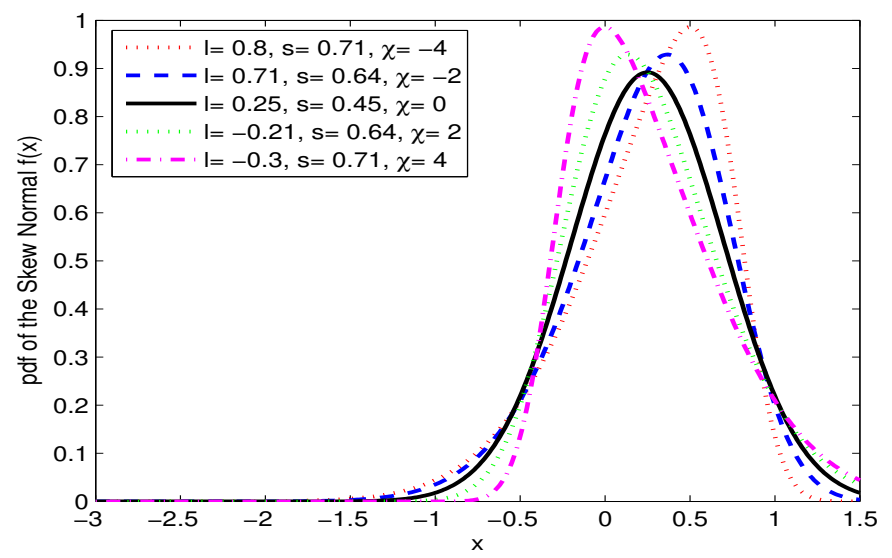

Figure 3: Skew-Normal probability distribution function

This graph represents the pdf $f_{X}$ of a Skew-Normal random variable X, for a range of parameters. $\chi$ is respectively equal to $-4,-2,0,2$ and 4 . Location and scale parameters $l$ and $s$ are calculated such that the first two moments are constant: the mean is $E[X]=0.25$ and the standard deviation is $\sigma(X)=0.2$.

Note that a high positive skewness parameter $\chi$ implies a fatter distribution of the gains (case when $\chi=4$ on Figure 3 ) and a very short tail of the losses. On the other hand, a high negative skewness parameter implies a fatter distribution of the losses as well as a short tail of the gains (case when $\chi=-4$ on Figure 3).

In the next paragraph, we illustrate the fact that the CPT-investor seeks a fat tail for the distribution of the gains and will thus prefer a high value for $\chi$.

\subsection{CPT-ratio of the Skew-normal distribution}

As we discussed in the previous section, the CPT-investor evaluates the performance of the distribution of a risky prospect using a generalized Omega performance measure $\Omega(\widetilde{y})$, as defined in equations (12) and (13), and also referred to as the CPT-ratio ${ }^{20}$. In this section, we focus on the sensitivity

\footnotetext{
${ }^{20} \mathrm{~A}$ mentioned upon definition of $\Omega(\widetilde{y})$, the latter refers to a generalized Omega measure that we call the CPT-ratio. The classical Omega measure at threshold level $\mathrm{L}$, for a cdf $\mathrm{F}$
} 
of $\Omega(\widetilde{y})$ to the different parameters of the distribution, such as the mean, the variance and the skewness, as well as the subjective characteristics of the CPT-investor given by the shape parameters $\alpha$ and $\beta$. Recall also that Proposition 4.1 states that a higher CPT-ratio implies a higher optimal holding in the risky asset. Thus this section also analyzes the sensitivity of the optimal holding to the different parameters of the model.

In Figure 4, we display the CPT-ratio $\Omega(\widetilde{y})$ as a function of the mean of the excess return, when the volatility and the skewness parameter $\chi$ are both fixed. Note that the location and scale parameters are adjusted so that the moments are fixed. Figure 4 displays intuitive effects on the $C P T$ ratio of changes in the mean, in the variance and in the skewness of the distribution. All curves of the left panel and the right panel are increasing, meaning that an increase in the mean implies an increase in $\Omega(\widetilde{y})$, ceteris paribus. From the left panel, one observes that when the volatility of the excess return increases, the CPT-ratio $\Omega(\widetilde{y})$ decreases. This is very intuitive and, in essence this means that the aforementioned generalized performance measure will increase when the reward increases (with no modification to the risk) or when the risk decreases (for a given average return). The right panel of Figure 4 shows that a higher skewness parameter $\chi$ leads to a higher value of the CPT-ratio $\Omega(\widetilde{y})$. This is consistent with the previous result of Barberis and Huang (2008) obtained with discrete distributions.

Let us now look at the effects of $\beta$ and $\alpha$ on the assessment of the risk by the CPT-investor. To do so, the CPT-ratio is displayed as a function of $1-\beta$ or $1-\alpha$. Figure 5 shows that $\Omega(\widetilde{y})$ is an increasing function of $1-\alpha$ and a decreasing function of $1-\beta$.

was denoted by $\bar{\Omega}_{F}(L)$, as in definition 4.1 . 

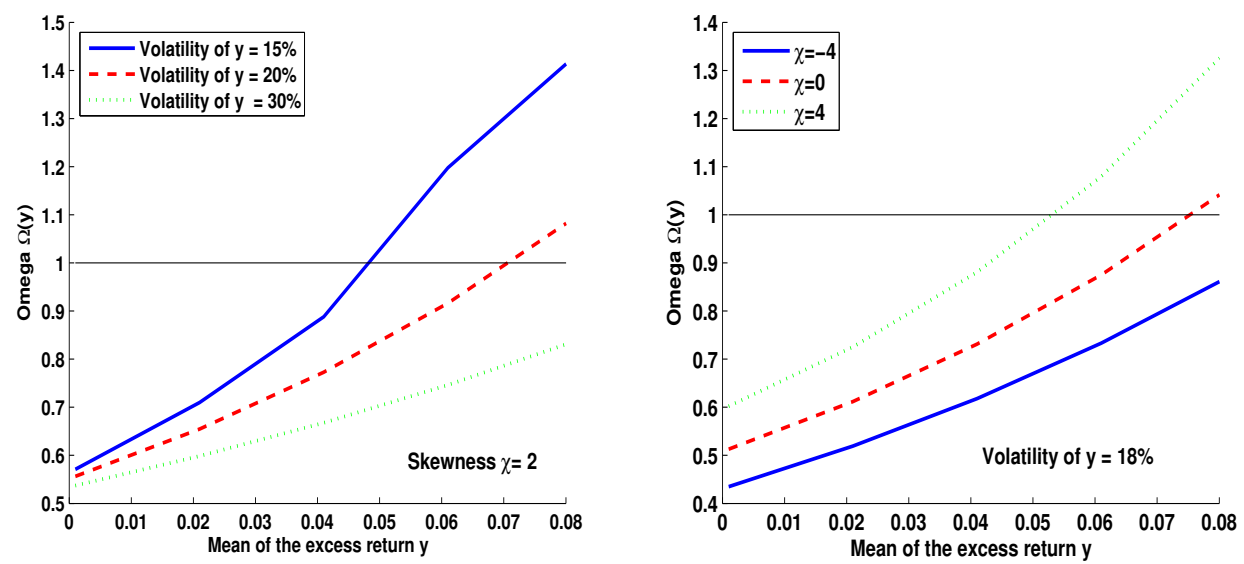

Figure 4: $\Omega(\widetilde{y})$ when $\widetilde{y}$ is Skew-normal

This graph represents the CPT-ratio $\Omega(\widetilde{y})$ of a Skew-Normal distribution with respect to the variance and the skewness parameter. In the left panel, the skewness parameter is equal to $\chi=2$ and the volatility takes the values $15 \%, 20 \%$ and $30 \%$. In the right panel, $\sigma=18 \%$ and the skewness parameter is equal to $-4,0$ and 4 . The other parameters are set to $\lambda=2.25, \gamma=.61, \delta=.69, \alpha=.8, \beta=.88$.

\subsection{Optimal portfolio: Plunging behavior}

Since we know how the CPT-ratio varies with respect to all of the parameters, it suffices to study the sensitivity of the optimal holding to the CPT-ratio. It turns out that the sensitivity to the CPT-ratio being slightly below 1 or slightly above 1 drastically changes the optimal portfolio of the CPT-investor. Indeed, Figure 6 displays the optimal holding as a function of $1-\alpha$ in the left panel, and as a function of $1-\beta$ in the right panel when $\Omega(\widetilde{y})$ is respectively $0.8,1$ and 1.2 .

Figure 4 and 5 both show that $\Omega(\widetilde{y})$ can sometimes exceed 1 and sometimes be lower than 1, for a realistic range for the parameters of the skewnormal distribution. The behavior of the CPT-investor will be extremely sensitive to changes in $\Omega(\widetilde{y})$, as can be seen from Figure 6 , and particularly so when $\alpha$ and $\beta$ are close to each other. We will take $\alpha=0.8$ and $\beta=0.88$. For this set of parameters, $1-\beta=0.12$ and $1-\alpha=0.2$, the left panel of the graph shows that the CPT-investor will invest almost all of his initial wealth in the risky asset if $\Omega(\widetilde{y})=1.2$, whereas he invests almost none of it in the 

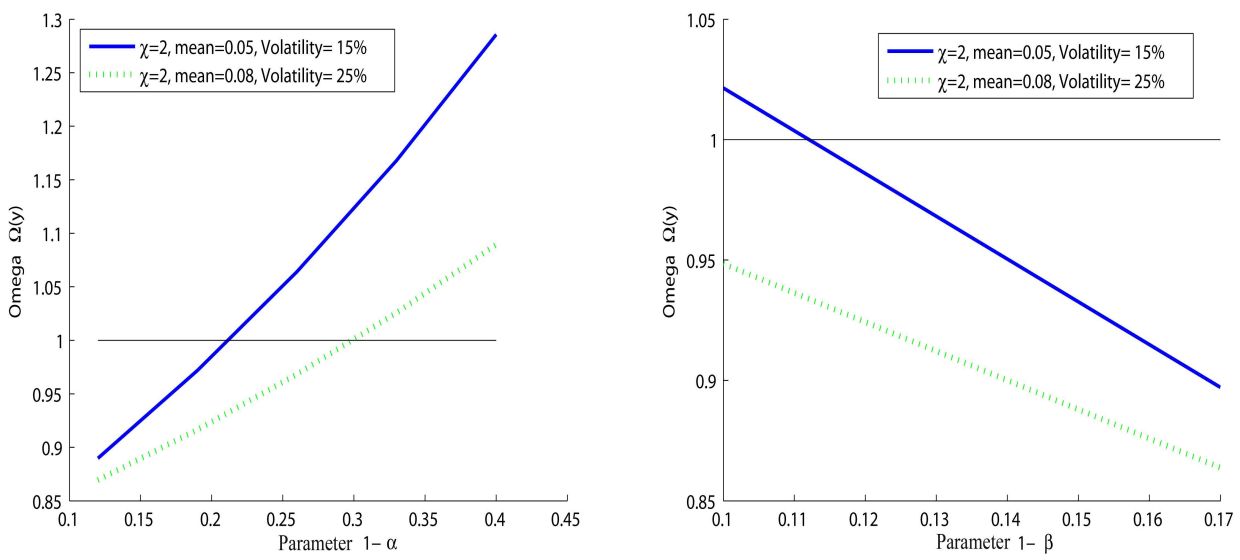

Figure 5: $\Omega(\widetilde{y})$ when $\widetilde{y}$ is Skew-normal

This graph represents the CPT-ratio $\Omega(\widetilde{y})$ of a Skew-Normal distribution with respect to $1-\alpha$ and to $1-\beta$ of the CPT-investor. In the left panel, $1-\beta=1-.88$ and $\alpha$ takes values between 0.6 and $\beta$. In the right panel, $\alpha=0.8$ and $\beta$ varies between $\alpha$ and 0.9. Other parameters are set to $\lambda=2.25, \gamma=0.61, \delta=0.69$. The skewness parameter is 2 , the excess return has a mean of $5 \%$ and a volatility of $15 \%$.

risky asset when $\Omega(\widetilde{y})=0.8$. Same effects can be seen from the right panel. When $\alpha$ is set to 0.8 and when $\beta$ is close to $\alpha, \zeta_{*}(\widetilde{y})$ is extremely sensitive to $\Omega(\widetilde{y})$. 

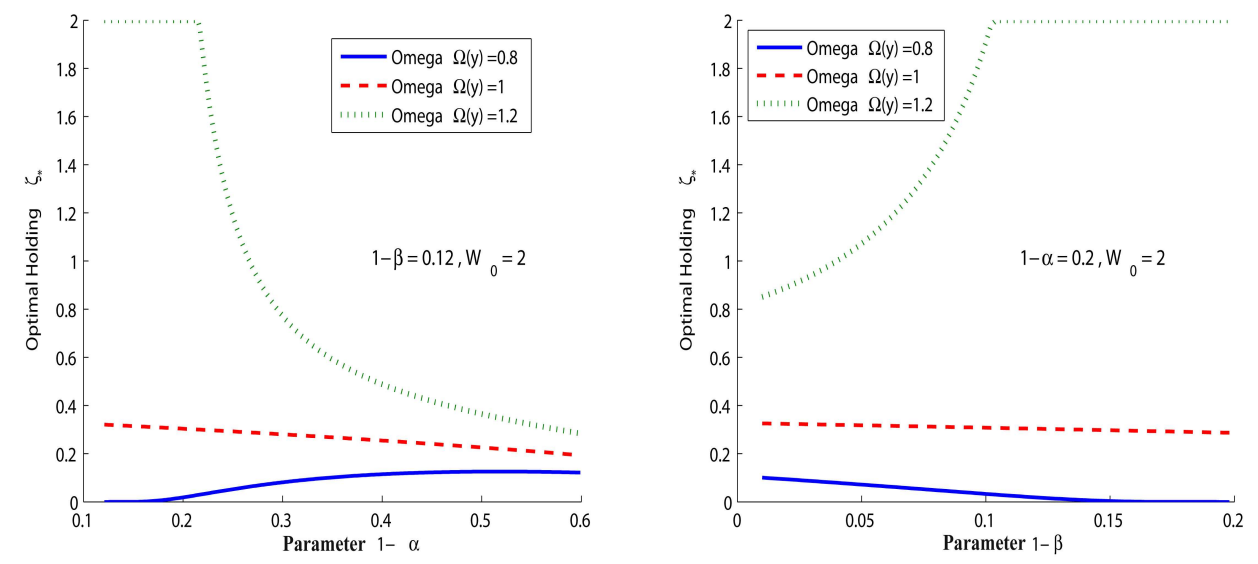

Figure 6: $\zeta_{*}(\widetilde{y})$ when $\widetilde{y}$ is Skew-normal

This graph represents the optimal holding in the risky asset as a function of $1-\beta$ and $1-\alpha$ for different possible values of the CPT-ratio $\Omega(\widetilde{y})$. In the left panel, $1-\beta=1-.88$ and $\alpha$ takes values between 0.4 and 0.88 . In the right panel, $\alpha=0.8$ and $\beta$ varies between $\alpha$ and 0.98 . The initial wealth is equal to $W_{0}=2$.

"Plunging" is used to describe the situation when the investor either invests everything safe or everything risky depending on the rate of return of the risky asset. In a sense, "plunging" is thus the absence of diversification. Using a two-state model, Schmidt and Zank (2007) discuss how it is sometimes optimal to diversify in Linear Cumulative Prospect Theory (LCPT) unlike in Dual Theory. However, in the presence of linear utilities, the optimal portfolio when the investor diversifies has a very specific composition. In our setting, the optimal holding could take any value between 0 and $W_{0}$. Nevertheless, Figure 6 shows that when $\alpha$ is close to $\beta$, the optimal holding is either close to 0 or to $W_{0}$, which means that the CPT-investor invests almost her full wealth or none of it in the risky asset. The CPT-investor is thus very sensitive to changes in the rate of return of the risky asset. These results show that behavior under CPT is more realistic than that of an economic agent behaving according to Dual Theory, or even that of a LCPT-investor (a CPT-investor with linear utilities, such as developed by Schmidt and Zank (2007)).

Figure 6 confirms Proposition 4.4. The ranking between CPT-ratios is preserved by the optimal holding $\zeta_{*}$. Indeed, this is a consequence of the fact that the three curves never intersect. For instance, the optimal holding in the risky asset when $\Omega(\widetilde{y})=1.2$ always stays above the optimal holding in the 
risky asset when $\Omega(\widetilde{y})=1$. However, this figure shows that the sensitivity of the optimal holding to $\alpha$ and $\beta$ is ambiguous. Indeed, $\Omega(\widetilde{y})$ is an increasing function of $1-\alpha$ whereas $\zeta_{*}$ could be an increasing function or a decreasing function of $1-\alpha$ or $1-\beta$.

\subsection{Skewness-Loving}

Next, we examine the effect of the skewness parameter $\chi$ on the CPT-ratio and on the optimal holding in the risky asset.

Proposition 5.1. Skewness Loving.

When the excess return on the risky asset has a skew-normal distribution, the higher the skewness the higher the CPT-investor's holding in the risky asset. In other words, if $\widetilde{y}$ has a skew-normal distribution with skewness parameter $\chi$, then when $\alpha<\beta$ we have $\frac{\partial \Omega(\widetilde{y})}{\partial \chi}>0$ where $\Omega(\widetilde{y})$ is the CPT-ratio defined in equation (12). Therefore:

$$
\frac{\partial \zeta_{*}(\widetilde{y})}{\partial \chi}>0
$$

Proof. The proof is given in the appendix.
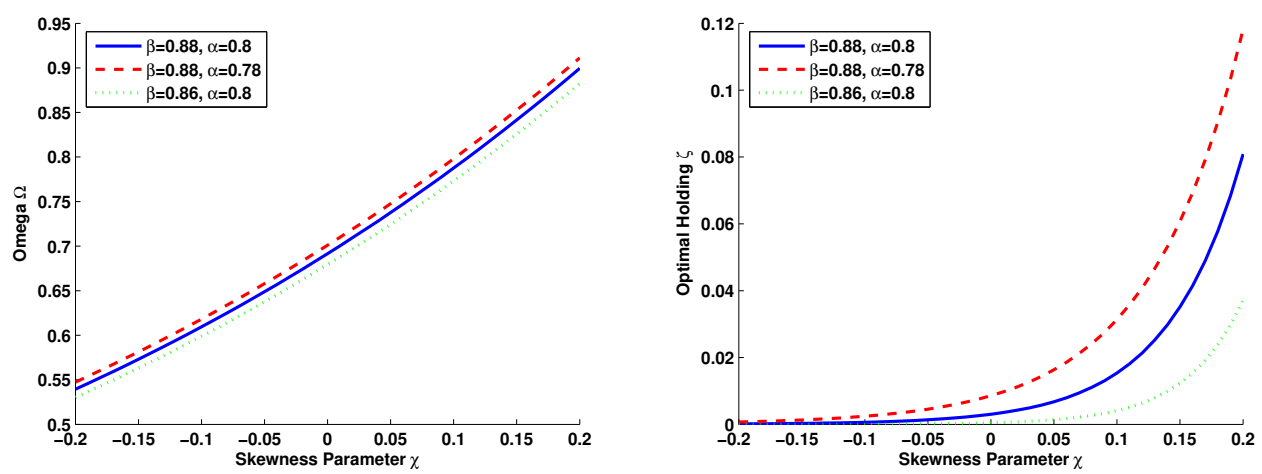

Figure 7: CPT-ratio $\Omega(\widetilde{y})$ and Optimal holding $\zeta_{*}$

This graph represents the CPT-ratio $\Omega(\widetilde{y})$ and the optimal holding $\zeta_{*}$ as a function of the parameter $\chi$ for different values of $\alpha$ and $\beta$. The other parameters were fixed to the following values: $\alpha=0.8, \beta=0.88, \lambda=2.25, \gamma=0.61, \delta=0.69, l=0.05$ and $s=0.1$. 
Figure 7 directly illustrates the Proposition 5.1.

However, we need to point out the simultaneous effects of $\chi$ on the mean and on the variance of the distribution of $\widetilde{y}$. Figure 8 displays the first two centered moments as a function of $\chi$. Their closed-form formulas are given in (30) and (31). Figure 8 shows that the expectation is increasing with the parameter $\chi$ which could also explain the fact that both $\Omega(\widetilde{y})$ and $\zeta_{*}$ are increasing with $\chi$.

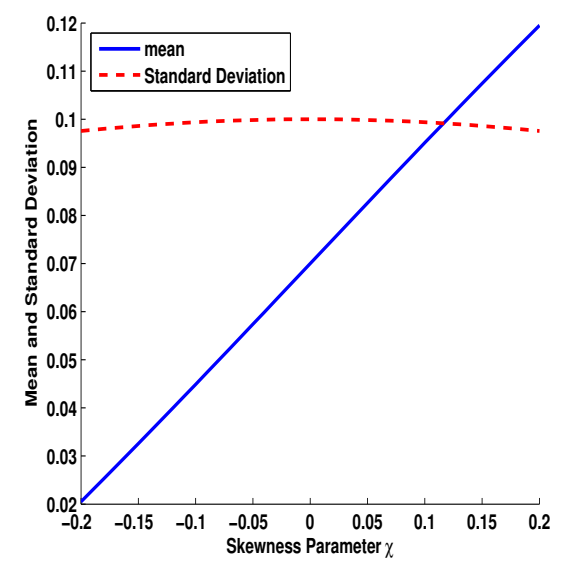

Figure 8: Mean and Variance as a function of the skewness parameter $\chi$

This graph represents the mean and the variance of a Skew-Normal distribution as a function of the skewness parameter $\chi$. The location and scale parameters are fixed and equal to $l=0.05$ and $s=0.1$, respectively.

To control these effects of the parameter $\chi$ on the first two moments, we have numerically solved for the skewness parameter $\chi$, the location parameter and the scale parameter such that the first two moments are constant, the mean being set to 0.07 and the volatility to $32 \%$. Figure 9 displays the values of $\Omega(\widetilde{y})$ and of the optimal holding $\zeta_{*}$ for a range of values of the skewness parameter, when the mean and the standard deviation are held constant.

Figure 9 shows that the CPT-investor still highly values skewness. This is consistent with the study of Barberis and Huang (2008). 

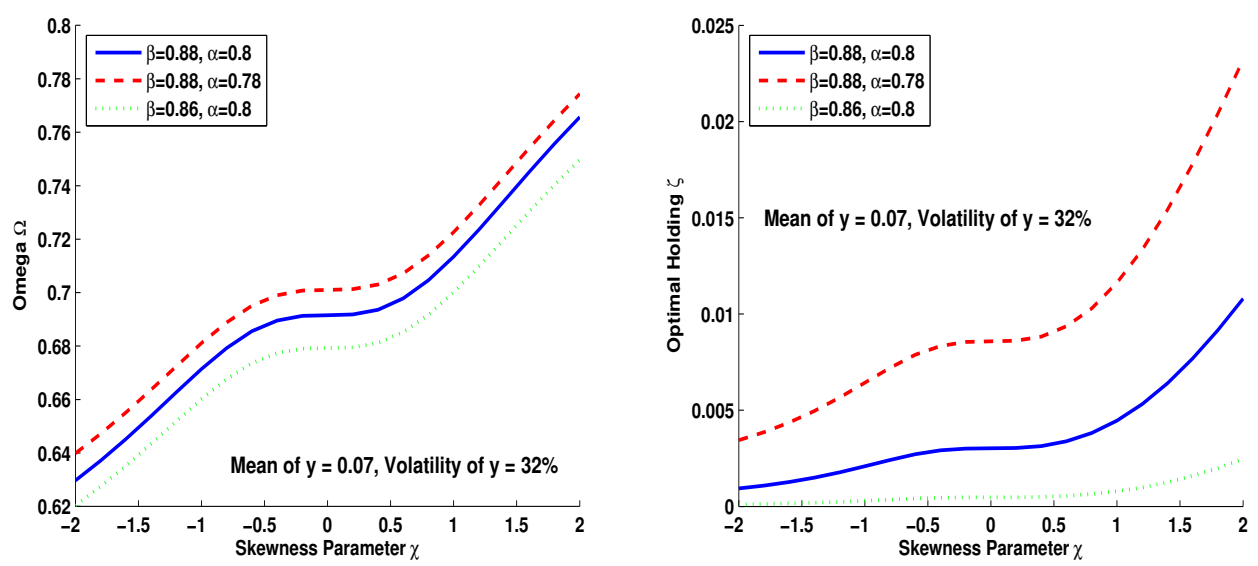

Figure 9: CPT-ratio $\Omega(\widetilde{y})$ and Optimal holding $\zeta_{*}$

This graph represents the CPT-ratio $\Omega(\widetilde{y})$ and the optimal holding $\zeta_{*}$ as a function of the parameter $\chi$ for different values of $\alpha$ and $\beta$. The location and scale parameters $l$ and $s$ are calculated such that the first two moments are constant. The mean is set to $E[X]=0.07$ and the standard deviation to $\sigma(X)=32 \%$.

\section{Summary and Conclusions}

This paper derives a closed-form expression of the optimal portfolio choice for an investor who behaves such as described by the Cumulative Prospect Theory (CPT) of Tversky and Kahneman (1992), under a specific choice of the status quo. When it is defined, the optimal holding in the risky asset for a CPT-investor is a function of a generalized Omega measure of the distribution of the excess return on the risky asset over the risk-free rate. Our results, however, depend on the choice of the status quo as the amount of wealth at the end of the period had all of the initial wealth been invested in the risk-free rate.

Therefore, adopting Cumulative Prospect Theory to study an economic agent's decision making seems to be consistent with adopting new performance measures. The fundamental idea is that losses and gains deserve a specific treatment that variance, for instance, fails to measure. If one believes that Omega is a better measure of the performance of a risky asset, then Cumulative Prospect Theory may certainly be a better model than Ex- 
pected Utility Theory to explain decision making. Indeed Omega measures are based on a separate evaluation of losses and gains. Hence, they are essentially focused on deviations from a benchmark level, consistently with the philosophy of Cumulative Prospect Theory. Our results confirm that CPTinvestors highly value skewness. However we would like to draw attention to the excessive sensitivity of the results to the parameter assumptions. A CPT-investor may suddenly switch his investment from almost fully invested in the risky asset to almost fully invested in the riskless asset for a slightly different set of parameters.

This is a first study. Further research will include the case of shifting reference levels, the case when there are several risky assets, so as to examine, for instance, the willingness of CPT-investors to diversify their portfolios, and the study of the effects of a zero-mean background risk correlated or not with the risky asset. 


\section{Appendix}

\section{A Mathematical background}

Let $(\Omega, \mathcal{F}, P)$ be a probability space and let $T:[0,1] \rightarrow[0,1]$ be a differentiable and increasing function with $T(0)=0$ and $T(1)=1$. In the following, let $\overline{\mathbb{R}}$ denote $\mathbb{R} \cup\{+\infty,-\infty\}$, and let $\overline{\mathbb{R}}^{+}$denote $\mathbb{R}^{+} \cup\{+\infty\}$.

Proposition A.1. The set function $\mu=T \circ P$ is a monotone set function called a distortion measure, with distortion $T$.

Proof. See Denneberg (1994).

Definition A.1. The distortion measure $T \circ P$ is a measure that is not necessarily additive, referred to as a capacity.

Definition A.2. A set function $\mu$ on a $\sigma$-algebra $\mathcal{S}$ of subsets of $\Omega$ is said to be finite if $\mu(A)<\infty$ for all $A \in \mathcal{S}$.

Definition A.3. Let $\mathcal{S}$ be a $\sigma$-algebra of subsets of $\Omega$, and let $A^{c}$ denote the complement of $A$. The conjugate on $\mathcal{S}$ of a finite set function $\mu$ on $\mathcal{S}$ is the set function $\bar{\mu}$ defined by: $\bar{\mu}(A):=\mu(\Omega)-\mu\left(A^{c}\right)$, for all $A \in \mathcal{S}$.

Note that we immediately have $\bar{\mu}(\Omega)=\mu(\Omega)$ and $\overline{\bar{\mu}}=\mu$.

Definition A.4. (See Schmeidler (1986) or Denneberg (1994))

For any nonnegative random variable $X$, the Choquet integral of $X$ with respect to the capacity $\mu=T \circ P$ is defined as:

$$
\int_{\Omega} X d \mu:=\int_{0}^{+\infty} \mu\{\omega \in \Omega: X(\omega) \geqslant y\} d y
$$

Definition A.5. For any nonnegative random variable $X$ with decumulative distribution function (ddf) $S_{X}$ under the probability measure $P$, for any capacity $\mu=T \circ P$, and for any increasing function $u: \overline{\mathbb{R}}^{+} \rightarrow \overline{\mathbb{R}}^{+}$, we define the functional $V_{\mu, u}(X)$ as the Choquet integral of $u(X)$ with respect to $\mu$ :

$$
\begin{aligned}
V_{\mu, u}(X) & =\int_{\Omega} u(X) d \mu=\int_{0}^{+\infty} \mu\{\omega \in \Omega: u(X(\omega)) \geqslant y\} d y \\
& =\int_{0}^{+\infty} T\left(S_{u(X)}(y)\right) d y
\end{aligned}
$$


Where $S_{u(X)}$ is the ddf of $u(X)$ under the probability measure $P$.

Proposition A.2. If the function $u$ is increasing, invertible and positively homogeneous of degree $k$, then the functional $V_{\mu, u}$ is also positively homogeneous of degree $k$.

Proof. Suppose $u: \overline{\mathbb{R}}^{+} \rightarrow \overline{\mathbb{R}}^{+}$is an increasing, invertible and positively homogeneous function of degree $\mathrm{k}$, so that $u(\alpha x)=\alpha^{k} u(x)$, for all $x \in \overline{\mathbb{R}}^{+}$ and for all $\alpha \in \mathbb{R}^{+}$. Then for all nonnegative random variables $Y: \Omega \rightarrow \overline{\mathbb{R}}^{+}$ and for all $\alpha \in \mathbb{R}^{+}$we have:

$$
\begin{aligned}
V_{\mu, u}(\alpha Y) & =\int_{0}^{+\infty} T\left[S_{u(\alpha Y)}(y)\right] d y=\int_{0}^{+\infty} T\left[S_{\alpha Y}(y)\right] d u(y) \\
& =\int_{0}^{+\infty} T\left[S_{Y}\left(\frac{y}{\alpha}\right)\right] d u(y)=\int_{0}^{+\infty} T\left[S_{Y}(x)\right] \alpha^{k} d u(x) \\
& =\alpha^{k} \int_{0}^{+\infty} T\left[S_{Y}(y)\right] d u(y)=\alpha^{k} V_{\mu, u}(Y)
\end{aligned}
$$

Which completes the proof.

Definition A.6. (The CPT-functional)

Let $u^{+}: \overline{\mathbb{R}}^{+} \rightarrow \overline{\mathbb{R}}^{+}$and $u^{-}: \overline{\mathbb{R}}^{+} \rightarrow \overline{\mathbb{R}}^{+}$be increasing, differentiable, invertible and concave functions with $u^{+}(0)=u^{-}(0)=0$.

Let $T^{+}:[0,1] \rightarrow[0,1]$ and $T^{-}:[0,1] \rightarrow[0,1]$ be differentiable and increasing functions with $T^{+}(0)=T^{-}(0)=0$ and $T^{+}(1)=T^{-}(1)=1$.

Let $\mu^{+}=T^{+} \circ P$ and $\mu^{-}=T^{-} \circ P$ be two capacities.

For any random variable $X:(\Omega, \mathcal{F}) \rightarrow(\overline{\mathbb{R}}, \mathcal{B}(\overline{\mathbb{R}}))$, define $X^{+}$and $X^{-}$as its positive and negative part, respectively. That is:

$$
X=X^{+}-X^{-}
$$

where $X^{+}=\max (X, 0)$ and $X^{-}=(-X)^{+}$. Then, we define the CPTfunctional $V^{c p t}(X)$ as:

$$
V^{c p t}(X)=V_{\mu^{+}, u^{+}}\left(X^{+}\right)+V_{\mu^{-}, u^{-}}\left(-X^{-}\right) .
$$


Proposition A.3. When $\mu^{-}$is finite, the CPT-functional can be written as:

$$
V^{c p t}(X)=V_{\mu^{+}, u^{+}}\left(X^{+}\right)-V_{\bar{\mu}^{-}, u^{-}}\left(X^{-}\right)
$$

Where $\bar{\mu}^{-}$is the conjugate of $\mu^{-}$. Furthermore, since by construction $X^{-}$is a nonnegative random variable, the CPT-functional is defined as:

$$
V^{c p t}(X)=V_{\mu^{+}, u^{+}}\left(X^{+}\right)-V_{\mu^{-}, u^{-}}\left(X^{-}\right)
$$

Proof. Denneberg (1994) proves the different parts of this result.

Proposition A.4. (An alternative form for the CPT-functional) When $T^{-} \circ P$ and $T^{+} \circ P$ are finite, equation (33) can be written as:

$$
V^{c p t}(X)=\int_{0}^{+\infty} T^{+}\left(S_{u^{+}\left(X^{+}\right)}(x)\right) d x-\int_{0}^{+\infty} T^{-}\left(S_{u^{-}\left(X^{-}\right)}(x)\right) d x
$$

or

$$
V^{c p t}(X)=\int_{0}^{+\infty} T^{+}\left(S_{X}(x)\right) d u^{+}(x)-\int_{0}^{+\infty} T^{-}\left(F_{X}(-x)\right) d u^{-}(x)
$$

or

$$
V^{c p t}(X)=\int_{0}^{+\infty} T^{+}\left(S_{X}(x)\right) d u^{+}(x)+\int_{-\infty}^{0} T^{-}\left(F_{X}(x)\right) d u^{-}(-x)
$$

Proof. - Equation (34) results directly from the definition of the Choquet integral $V_{\mu, u}$, since $T^{-} \circ P$ and $T^{+} \circ P$ are finite.

- Since $u^{+}$is invertible and increasing, its inverse $\left(u^{+}\right)^{-1}$ exists and is increasing. Therefore, $\forall x \in \mathbb{R}, P\left[u^{+}(X) \geqslant x\right]=P\left[X \geqslant\left(u^{+}\right)^{-1}(x)\right]$. Hence using the change of variable $z=\left(u^{+}\right)^{-1}(x)$ so that $x=u^{+}(z)$ yields:

$$
V_{\mu^{+}, u^{+}}(X)=\int_{0}^{+\infty} T^{+}\left(S_{X}(z)\right) d u^{+}(z)
$$

Now, $\forall x \in \mathbb{R}^{+}(X(\omega) \geqslant x) \Rightarrow\left(X^{+}(\omega) \geqslant x\right)$. 
Thus, $\forall x \in \mathbb{R}^{+} S_{X}(x)=S_{X^{+}}(x)$, so that:

$V_{\mu^{+}, u^{+}}(X)=\int_{0}^{+\infty} T^{+}\left(S_{X}(z)\right) d u^{+}(z)=\int_{0}^{+\infty} T^{+}\left(S_{X^{+}}(z)\right) d u^{+}(z)=V_{\mu^{+}, u^{+}}\left(X^{+}\right)$

Similarly, using the change of variable $z=\left(u^{-}\right)^{-1}(x)$,

$$
V_{\mu^{-}, u^{-}}(X)=\int_{0}^{+\infty} T^{-}\left(S_{u^{-}(X)}(x)\right) d x=\int_{0}^{+\infty} T^{-}\left(S_{X^{-}}(z)\right) d u^{-}(z)
$$

Now, $\forall x \in \mathbb{R}^{+}\left(X^{-}(\omega) \geqslant x\right) \Rightarrow(X(\omega) \leqslant-x)$.

Thus, $\forall x \in \mathbb{R}^{+} S_{X^{-}}(x)=F_{X}(-x)$ where $F_{X}$ is the cdf of X. Hence:

$$
V_{\mu^{-}, u^{-}}(X)=\int_{0}^{+\infty} T^{-}\left(F_{X}(-z)\right) d u^{-}(z)
$$

Therefore:

$$
\begin{aligned}
V^{c p t}(X) & =V_{\mu^{+}, u^{+}}\left(X^{+}\right)-V_{\mu^{-}, u^{-}}\left(X^{-}\right) \\
& =\int_{0}^{+\infty} T^{+}\left(S_{X}(x)\right) d u^{+}(x)-\int_{0}^{+\infty} T^{-}\left(F_{X}(-x)\right) d u^{-}(x)
\end{aligned}
$$

- Equation (36) results directly from equation (35) by a simple change of variable, which completes the proof.

\section{B Proofs}

\section{B.1 Derivation of equation (9)}

Proof. Let $D=\zeta \widetilde{y}$, where $\zeta>0$ (no short-selling). From equation (35) or equation (7), we have:

$$
V^{c p t}(D)=\int_{0}^{+\infty} T^{+}\left(S_{D}(x)\right) d u^{+}(x)-\int_{0}^{+\infty} T^{-}\left(F_{D}(-x)\right) d u^{-}(x)
$$


Furthermore, $S_{D}(x)=S_{\widetilde{y}}\left(\frac{x}{\zeta}\right)$ and $F_{D}(x)=F_{\widetilde{y}}\left(\frac{x}{\zeta}\right)$. Then one obtains:

$$
\int_{0}^{+\infty} T^{+}\left(S_{D}(x)\right) d u^{+}(x)=\int_{0}^{+\infty} T^{+}\left[S_{\widetilde{y}}\left(\frac{x}{\zeta}\right)\right] \alpha x^{\alpha-1} d x
$$

Letting $y=\frac{x}{\zeta}$, so that $x=y \zeta$ and $d x=\zeta d y$ yields:

$$
\begin{aligned}
\int_{0}^{+\infty} T^{+}\left(S_{D}(x)\right) d u^{+}(x) & =\int_{0}^{+\infty} T^{+}\left[S_{\widetilde{y}}(y)\right] \alpha y^{\alpha-1} \zeta^{\alpha-1} \zeta d y \\
& =\zeta^{\alpha} \int_{0}^{+\infty} T^{+}\left[S_{\widetilde{y}}(y)\right] d u^{+}(y)
\end{aligned}
$$

Similarly, we have:

$$
\begin{aligned}
\int_{0}^{+\infty} T^{-}\left(F_{D}(-x)\right) d u^{-}(x) & =\int_{0}^{+\infty} T^{-}\left(F_{\widetilde{y}}\left(-\frac{x}{\zeta}\right)\right) \lambda \beta x^{\beta-1} d x \\
\int_{0}^{+\infty} T^{-}\left(F_{D}(-x)\right) d u^{-}(x) & =\int_{0}^{+\infty} T^{-}\left(F_{\widetilde{y}}(-y)\right) \lambda \beta \zeta^{\beta-1} y^{\beta-1} \zeta d y \\
& =\zeta^{\beta} \int_{0}^{+\infty} T^{-}\left(F_{\widetilde{y}}(-y)\right) d u^{-}(y)
\end{aligned}
$$

Consequently, the CPT-objective function of the end-of-period deviation from the reference level of wealth can be written as:

$$
V^{c p t}(D)=\zeta^{\alpha} \int_{0}^{+\infty} T^{+}\left[S_{\widetilde{y}}(y)\right] d u^{+}(y)-\zeta^{\beta} \int_{0}^{+\infty} T^{-}\left(F_{\widetilde{y}}(-y)\right) d u^{-}(y)
$$

Note that the result also holds for $\zeta=0$ since $D=\zeta \widetilde{y}$ and $V^{c p t}(0)=0$.

\section{B.2 Proof of proposition 4.2}

Proof. The CPT-ratio is given by $\Omega(\widetilde{y})=\frac{\int_{0}^{+\infty} T^{+}\left[S_{\widetilde{y}}(y)\right] d u^{+}(y)}{\int_{0}^{+\infty} T^{-}\left[F_{\widetilde{y}}(-y)\right] d u^{-}(y)}$. Therefore, for any $m>0$, we have:

$$
\Omega(m \widetilde{y})=\frac{\int_{0}^{+\infty} T^{+}\left[S_{m \widetilde{y}}(y)\right] d u^{+}(y)}{\int_{0}^{+\infty} T^{-}\left[F_{m \widetilde{y}}(-y)\right] d u^{-}(y)}=\frac{A(m)}{B(m)}
$$


where:

$$
\left\{\begin{array}{l}
A(m)=\int_{0}^{+\infty} T^{+}\left[S_{m \widetilde{y}}(y)\right] d u^{+}(y)=\int_{0}^{+\infty} T^{+}\left[S_{\widetilde{y}}\left(\frac{y}{m}\right)\right] d u^{+}(y) \\
B(m)=\int_{0}^{+\infty} T^{-}\left[F_{m \widetilde{y}}(-y)\right] d u^{-}(y)=\int_{0}^{+\infty} T^{-}\left[F_{\widetilde{y}}\left(-\frac{y}{m}\right)\right] d u^{-}(y)
\end{array}\right.
$$

Letting $x=\frac{y}{m}$, so that $d u^{+}(y)=m^{\alpha} d u^{+}(x)$ and $d u^{-}(y)=m^{\beta} d u^{-}(x)$, yields:

$$
\left\{\begin{array}{l}
A(m)=m^{\alpha} \int_{0}^{+\infty} T^{+}\left[S_{\widetilde{y}}(x)\right] d u^{+}(x) \\
B(m)=m^{\beta} \int_{0}^{+\infty} T^{-}\left[F_{\widetilde{y}}(-x)\right] d u^{-}(x)
\end{array}\right.
$$

Consequently:

$$
\Omega(m \widetilde{y})=m^{\alpha-\beta}\left[\frac{\int_{0}^{+\infty} T^{+}\left[S_{\widetilde{y}}(y)\right] d u^{+}(y)}{\int_{0}^{+\infty} T^{-}\left[F_{\widetilde{y}}(-y)\right] d u^{-}(y)}\right]=m^{\alpha-\beta} \Omega(\widetilde{y})
$$

Which completes the proof.

\section{B.3 Proof of proposition 5.1}

Proof. We consider the case when $\widetilde{y}$ is a skew-normal random variable. The case when $\widetilde{y}$ is a shifted skew-normal random variable is a straightforward consequence. Let $\widetilde{y}$ be a skew-normal random variable with skewness parameter $\chi$. We write $\widetilde{y} \sim S N(\chi)$. Then the pdf $f_{\widetilde{y}}$ and the cdf $F_{\widetilde{y}}$ of $\widetilde{y}$ are given by:

$$
\left\{\begin{array}{l}
f_{\widetilde{y}}(y ; \chi)=2 \phi(y) \Phi(\chi y) \\
F_{\widetilde{y}}(y ; \chi)=2 \int_{-\infty}^{y} \int_{-\infty}^{\chi s} \phi(s) \phi(t) d t d s
\end{array}\right.
$$

where $\phi$ and $\Phi$ are respectively the pdf and cdf of the standard normal distribution. 
Hence:

$$
\begin{aligned}
\frac{\partial F_{\widetilde{y}}(y ; \chi)}{\partial \chi} & =2 \frac{\partial}{\partial \chi} \int_{-\infty}^{y} \int_{-\infty}^{\chi s} \phi(s) \phi(t) d t d s \\
& =2 \int_{-\infty}^{y} \phi(s) \frac{\partial}{\partial \chi}\left[\int_{-\infty}^{\chi s} \phi(t) d t\right] d s \\
& =2 \int_{-\infty}^{y} s \phi(s) \phi(\chi s) d s
\end{aligned}
$$

Now, $\phi(s)=\frac{1}{\sqrt{2 \pi}} e^{-\frac{1}{2} s^{2}}$ and $\phi(\chi s)=\frac{1}{\sqrt{2 \pi}} e^{-\frac{1}{2} s^{2} \chi^{2}}$. Therefore:

$$
\phi(s) \phi(\chi s)=\left(\frac{1}{\sqrt{2 \pi}}\right)^{2} e^{-\frac{1}{2} s^{2}\left(1+\chi^{2}\right)}
$$

Thus:

$$
\frac{\partial F_{\widetilde{y}}(y ; \chi)}{\partial \chi}=\frac{2}{\sqrt{2 \pi}} \int_{-\infty}^{y} \frac{s}{\sqrt{2 \pi}} e^{-\frac{1}{2} s^{2}\left(1+\chi^{2}\right)} d s
$$

Letting $u=s \sqrt{1+\chi^{2}}$ yields:

$$
\frac{\partial F_{\widetilde{y}}(y ; \chi)}{\partial \chi}=-\frac{1}{\pi} \frac{1}{1+\chi^{2}} e^{-\frac{1}{2} y^{2}\left(1+\chi^{2}\right)}
$$

Therefore,

$$
\forall y \in \mathbb{R}, \quad \frac{\partial F_{\widetilde{y}}(y ; \chi)}{\partial \chi}<0
$$

The CPT-ratio is given by equation (12) as:

$$
\Omega(\widetilde{y})=\frac{\int_{0}^{+\infty} T^{+}\left[S_{\widetilde{y}}(y)\right] d u^{+}(y)}{\int_{0}^{+\infty} T^{-}\left[F_{\widetilde{y}}(-y)\right] d u^{-}(y)}=\frac{A(\chi)}{B(\chi)}
$$

Consequently,

$$
\frac{\partial \Omega(\widetilde{y})}{\partial \chi}=\frac{B(\chi) \frac{\partial A(\chi)}{\partial \chi}-A(\chi) \frac{\partial B(\chi)}{\partial \chi}}{B^{2}(\chi)}
$$

The sign of $\frac{\partial \Omega(\widetilde{y})}{\partial \chi}$ depends on that of $B(\chi) \frac{\partial A(\chi)}{\partial \chi}-A(\chi) \frac{\partial B(\chi)}{\partial \chi}$. 


$$
\begin{aligned}
\frac{\partial A(\chi)}{\partial \chi} & =\int_{0}^{+\infty} \frac{\partial}{\partial \chi}\left(T^{+}\left[S_{\widetilde{y}}(y)\right]\right) d u^{+}(y) \\
& =\int_{0}^{+\infty}\left(\frac{\partial T^{+}\left[S_{\widetilde{y}}(y)\right]}{\partial S_{\widetilde{y}}(y)}\right)\left(\frac{\partial S_{\widetilde{y}}(y)}{\partial \chi}\right) d u^{+}(y) \\
& =-\int_{0}^{+\infty}\left(\frac{\partial T^{+}\left[S_{\widetilde{y}}(y)\right]}{\partial S_{\widetilde{y}}(y)}\right)\left(\frac{\partial F_{\widetilde{y}}(y)}{\partial \chi}\right) d u^{+}(y) \\
& >0
\end{aligned}
$$

using equation (39), the fact that $T^{+}$is a monotone increasing function, and the fact that $u^{+}: \overline{\mathbb{R}}^{+} \rightarrow \overline{\mathbb{R}}^{+}$.

Similarly,

$$
\begin{aligned}
\frac{\partial B(\chi)}{\partial \chi} & =\int_{0}^{+\infty} \frac{\partial}{\partial \chi}\left(T^{-}\left[F_{\widetilde{y}}(-y)\right]\right) d u^{-}(y) \\
& =\int_{0}^{+\infty}\left(\frac{\partial T^{-}\left[F_{\widetilde{y}}(-y)\right]}{\partial F_{\widetilde{y}}(-y)}\right)\left(\frac{\partial F_{\widetilde{y}}(-y)}{\partial \chi}\right) d u^{-}(y) \\
& <0
\end{aligned}
$$

using equation (39), the fact that $T^{-}$is a monotone increasing function, and the fact that $u^{-}: \overline{\mathbb{R}}^{+} \rightarrow \overline{\mathbb{R}}^{+}$. Thus $A(\chi)>0, \frac{\partial A(\chi)}{\partial \chi}>0, B(\chi)>0, \frac{\partial B(\chi)}{\partial \chi}<0$. Hence $B(\chi) \frac{\partial A(\chi)}{\partial \chi}-A(\chi) \frac{\partial B(\chi)}{\partial \chi}>0$. Thus, $\frac{\partial \Omega(\widetilde{y})}{\partial \chi}>0$ and one obtains $\frac{\partial \zeta_{*}}{\partial \chi}>0$. Since $\zeta_{*}=\left[\Omega(\widetilde{y})\left(\frac{\alpha}{\beta}\right)\right]^{\beta-\alpha}$ when $\alpha<\beta$.

$$
\frac{\partial \zeta_{*}}{\partial \chi}>0 \text {. }
$$




\section{References}

Abdellaoui, M., H. Bleichrodt, and C. Paraschiv (2007): "Loss Aversion Under Prospect Thepry: A Parameter-Free Measurement," Management Science, 53(10), 1659-1674.

Allais, M. (1953): "Le Comportement de l'Homme Rationnel Devant le Risque: Critique des Axiomes et Postulats de l'École Américaine," Econometrica, 21(4), 503-546.

Barberis, N., AND M. Huang (2008): "Stocks as Lotteries: The Implications of Probability Weighting for Security Prices," American Economic Review, 98(5), 2066-2100.

Baucells, M., and F. Heukamp (2006): "Stochastic dominance and cumulative theory," Management Science, 52(9), 1409-1423.

Benartzi, S., And R. Thaler (1995): "Myopic Loss Aversion and the Equity Premium Puzzle," The Quarterly Journal of Economics, 110(1), $73-92$.

Berkelaar, A., R. Kouwenberg, and T. Post (2004): "Optimal Portfolio Choice Under Loss Aversion," The Review of Economics and Statistics, 86(4), 973-987.

Bernardo, A., And O. Ledoit (2000): "Gain, Loss and Asset Pricing," Journal of Political Economy, 108(1), 144-172.

Camerer, C., G. Loewenstein, and M. Rabin (2004): Advances in Behavioral Economics. Princeton University Press.

Cascon, A., C. Keating, and W. Shadwick (2003): "The Omega Function," The Finance Development Centre, Working Paper.

Davies, G. B., and S. E. Satchell (2007): "The Behavioural Components of Risk Aversion," Journal of Mathematical Psychology, 51(1), 1-13.

De Giorgi, E., And T. Hens (2006): "Making Prospect Theory Fit for Finance," Financial Markets and Portfolio Management, 20(3), 339-360.

De Giorgi, E., T. Hens, And H. Levy (2004): "Existence of CAPM Equilibria with Prospect Theory Preferences," NCCR-FINRISK Working Paper, no. 85, Available at SSRN: http://ssrn.com/abstract=420184. 
De Giorgi, E., T. Hens, And J. Mayer (2006): "A Behavioral Foundation of Reward-Risk Portfolio Selection and the Asset Allocation Puzzle," EFA 2006 Zurich Meetings Paper, Available at SSRN: http://ssrn.com/abstract=899273.

De Giorgi, E., T. Hens, And M. Rieger (2008): "Financial Market Equilibria with Cumulative Prospect Theory," Swiss Finance Institute Research Paper, no. 07-21, Available at SSRN: http://ssrn.com/abstract=985539.

DenneberG, D. (1994): Non-Additive Measure and Integral. Kluwer Academic Publishers.

EdWARds, W. (1962): "Subjective Probabilities Inferred from Decisions," Psychological Review, 69(2), 109-135.

Eeckhoudt, L., C. Gollier, and H. Schlesinger (2005): Economic and Financial Decisions under Risk. Princeton University Press.

EllsberG, D. (1961): "Risk, Ambiguity and the Savage Axioms," Quarterly Journal of Economics, 75(4), 643-669.

Fellner, W. (1961): "Distortion of Subjective Probabilities as a Reaction to Uncertainty," Quarterly Journal of Economics, 75(4), 670-689.

Fishburn, P. (1988): Nonlinear Preference and Utility Theory. The Johns Hopkins University Press.

Genton, M. (2004): Skew-Elliptical Distributions and Their Applications: A Journey Beyond Normality. Chapman and Hall/CRC.

Gollier, C. (1996): "Optimum Insurance of Approximate Losses," Journal of Risk and Insurance, 63(3), 369-380.

- (2001): The Economics of Risk and Time. The MIT Press.

Gomes, F. (2005): "Portfolio Choice and Trading Volume with Loss-Averse Investors," Journal of Business, 78(2), 675-706.

HandA, J. (1977): "Risk, Probabilities and a New Theory of Cardinal Utility," Journal of Political Economy, 85(1), 97-122.

Jarrow, R., And F. Zhao (2006): "Downside Loss Aversion and Portfolio Management," Management Science, 52(4), 558-566. 
Jin, H., And X. Y. Zhou (2008): "Behavioral Portfolio Selection in Continous Time," Mathematical Finance, 18(3), 385-426.

Kahneman, D., And A. Tversky (1979): "Prospect Theory: An Analysis of Decision Under Risk," Econometrica, 47(2), 263-291.

Keating, C., and W. Shadwick (2002): "A Universal Performance Measure," Journal of Performance Measurement, 6(3), 59-84.

Köbberling, V., And P. Wakker (2005): "An Index of Loss Aversion," Journal of Economic Theory, 122(1), 119-131.

Merton, R. (1969): "Lifetime Portfolio Selection under Uncertainty: The Continuous-Time Case," The Review of Economics and Statistics, 51(3), $247-257$.

NeILson, W. S. (2002): "Comparative Risk Sensitivity with ReferenceDependent Preferences," The Journal of Risk and Uncertainty, 24(2), 131142.

Quigain, J. (1982): "A Theory of Anticipated Utility," Journal of Economic Behavior, 3(4), 323-343.

Schmeidler, D. (1986): "Integral Representation without Additivity," Proceedings of the American Mathematical Society, 97(2), 255-261.

- (1989): "Subjective Probability and Expected Utility without Additivity," Econometrica, 57(3), 571-587.

Schmidt, U., And H. ZAnK (2005): "What is Loss Aversion?," The Journal of Risk and Uncertainty, 30(2), 157-167.

_ (2007): "Linear Cumulative Prospect Theory with Applications to Portfolio Selection and Insurance Demand," Decisions in Economics and Finance, 30, 1-18.

Starmer, C. (2000): "Developments in Non-Expected Utility Theory: The Hunt for a Descriptive Theory of Choice under Risk," Journal of Economic Literature, 38(2), 332-382.

Tversky, A., And D. Kahneman (1992): "Advances in Prospect Theory: Cumulative Representation of Uncertainty," The Journal of Risk and Uncertainty, 5(4), 297-323. 
Wakker, P. (1994): "Separating Marginal Utility and Probabilistic Risk Aversion," Theory and Decision, 36(1), 1-44.

Wakker, P., And A. Tversky (1993): "An Axiomatization of Cumulative Prospect Theory," The Journal of Risk and Uncertainty, 7(7), 147-176.

Wang, S., And V. Young (1998): "Ordering Risks: Expected Utility Theory versus Yaari's Dual Theory of Risk," Insurance: Mathematics and Economics, 22, 145-161.

YAARI, M. (1987): "The Dual Theory of Choice under Risk," Econometrica, 55(1), 95-115.

Zank, H. (2009): "On Probabilities and Loss Aversion," Theory and Decision - Forthecoming. 